February 25, 2019

\title{
POISSON-COMMUTATIVE SUBALGEBRAS AND COMPLETE INTEGRABILITY ON NON-REGULAR COADJOINT ORBITS AND FLAG VARIETIES
}

\author{
DMITRI I. PANYUSHEV AND OKSANA S. YAKIMOVA \\ To the memory of Bertram Kostant
}

\begin{abstract}
The purpose of this paper is to bring together various loose ends in the theory of integrable systems. For a semisimple Lie algebra $\mathfrak{g}$, we obtain several results on completeness of homogeneous Poisson-commutative subalgebras of $\mathcal{S}(\mathfrak{g})$ on coadjoint orbits. This concerns, in particular, Mishchenko-Fomenko and Gelfand-Tsetlin subalgebras.
\end{abstract}

\section{INTRODUCTION}

Symplectic manifolds or varieties $(M, \omega)$ provide a natural setting for integrable systems. The algebra of "suitable" functions on $M, \operatorname{Fun}(M)$, carries a Poisson bracket, and connections with Geometric Representation Theory occur if a Hamiltonian action of a Lie group $Q$ on $M$ is given. Let $\mu: M \rightarrow \mathfrak{q}^{*}=(\operatorname{Lie} Q)^{*}$ be the corresponding moment mapping and $\mathcal{S}(\mathfrak{q})$ the symmetric algebra of $\mathfrak{q}$. Then $\mathcal{S}(\mathfrak{q})$ is a Poisson algebra and the co-morphism $\mu^{*}: \mathcal{S}(\mathfrak{q}) \rightarrow \operatorname{Fun}(M)$ is a Poisson homomorphism. Therefore, if $\mathcal{A} \subset \mathcal{S}(\mathfrak{q})$ is Poissoncommutative, then so is $\mu^{*}(\mathcal{A})$. For a coisotropic Hamiltonian action $(Q, M)$, one obtains a completely integrable system on $M$, see [VY18]. The key point here is the existence of a Poisson-commutative algebra $\mathcal{A} \subset \mathcal{S}(\mathfrak{q})$ that is complete, i.e., it provides a complete family in involution on a generic $Q$-orbit in the image of $\mu$, see Definition 1.

Two most celebrated examples of Poisson-commutative subalgebras are the GelfandTsetlin subalgebras of $\mathcal{S}\left(\mathfrak{s l}_{n}\right)$ and $\mathcal{S}\left(\mathfrak{s o}_{n}\right)$. Their definition goes back to [GT50, GT50', GS83, GS83']. The success of that construction heavily relies on the existence of chains of coisotropic actions. We prove that both these algebras are complete on every coadjoint orbit. For arbitrary simple Lie algebras $\mathfrak{g}$, a large supply of Poisson-commutative subalgebras of $\mathcal{S}(\mathfrak{g})$ is given by the argument shift method, see below.

Our ground field $\mathbb{k}$ is algebraically closed and of characteristic 0 . Let $G$ be a reductive algebraic group over $\mathbb{k}$ with $\mathfrak{g}=\operatorname{Lie} G$. Poisson-commutative subalgebras of $\mathcal{S}(\mathfrak{g})$ attract a great deal of attention, because of their relationship to geometric representation theory.

2010 Mathematics Subject Classification. 17B63, 14L30, 17B08, 17B20, 22E46.

Key words and phrases. integrable systems, moment map, coisotropic actions, coadjoint orbits.

The research of the first author was supported by the Russian Foundation for Sciences. The second author is funded by the Deutsche Forschungsgemeinschaft (DFG, German Research Foundation) — project number 330450448 . 
If $\mathcal{A} \subset \mathcal{S}(\mathfrak{g})$ is Poisson-commutative, then $\operatorname{tr} \operatorname{deg} \mathcal{A} \leqslant \boldsymbol{b}(\mathfrak{g})=\frac{1}{2}(\operatorname{dim} \mathfrak{g}+\mathrm{rk} \mathfrak{g})$. This is the dimension of a Borel subalgebra of $\mathfrak{g}$. (For arbitrary Lie algebras $\mathfrak{q}$, the rank should be replaced with the index, ind q.) In [MF78], a certain Poisson-commutative subalgebra $\mathcal{F}_{a} \subset \mathcal{S}(\mathfrak{g})$ is constructed for any $a \in \mathfrak{g}^{*}$. Following [Vi91], we say that $\mathcal{F}_{a}$ is the MishchenkoFomenko subalgebra (associated with a) or just an MF-subalgebra. Say that $a \in \mathfrak{g}^{*}$ is regular if $\operatorname{dim}(G a)=\operatorname{dim} \mathfrak{g}-\mathrm{rk} \mathfrak{g}$ and write $\mathfrak{g}_{\text {reg }}^{*}$ for the set of regular elements. It is known that $\operatorname{tr} \operatorname{deg} \mathcal{F}_{a}=\boldsymbol{b}(\mathfrak{g})$ if and only if $a \in \mathfrak{g}_{\mathrm{reg}}^{*}$. The importance of MF-subalgebras and their quantum counterparts is advocated e.g. in [FFR10, Vi91, K09].

We prove that, for any $a \in \mathfrak{g}_{\text {reg }}^{*}, \mathcal{F}_{a}$ is complete on each regular and each closed $G$-orbit (Theorem 2.4). The closed orbits are of extreme importance in view of their connection with flag varieties and integrable systems related to the compact form of $\mathfrak{g}$.

The crucial rôle of nilpotent $G$-orbits is seen in the observation that if an arbitrary homogeneous Poisson-commutative subalgebra of $\mathcal{S}(\mathfrak{g})$ is complete on any nilpotent orbit, then it is complete on every orbit, see Proposition 2.5 and Corollary 2.6. This implies that there is a dense open subset $U \subset \mathfrak{g}_{\text {reg }}^{*}$ such that $\mathcal{F}_{a}(a \in U)$ is complete on every $G$-orbit, see Proposition 2.8. Another striking feature is that the question of completeness on regular orbits is reduced to the unique regular nilpotent orbit.

The starting point of the Gelfand and Tsetlin construction [GT50, GT50'] for $\mathfrak{g}=\mathfrak{s l}_{n}$ or $\mathfrak{s o}_{n}$, is a chain of Lie algebras

$$
\mathfrak{g}=\mathfrak{g}(n) \supset \mathfrak{g}(n-1) \supset \cdots \supset \mathfrak{g}(1),
$$

where $\mathfrak{g}(k)=\mathfrak{s l}_{k}$ or $\mathfrak{s o}_{k}$. The Gelfand-Tsetlin $(=G T)$ subalgebra $\hat{\mathcal{C}}$ of the enveloping algebra $\mathcal{U}(\mathfrak{g})$ is generated by the centres of $\mathcal{U}(\mathfrak{g}(k))$ with $1 \leqslant k \leqslant n$. Then $\mathcal{C}:=\operatorname{gr}(\hat{\mathcal{C}})$ is a Poissoncommutative subalgebra of $\mathcal{S}(\mathfrak{g})$ with tr.deg $\mathcal{C}=\boldsymbol{b}(\mathfrak{g})$. The main reason behind many nice features of the GT-subalgebras $\mathcal{C}$ is that $\left(\mathrm{GL}_{n}, \mathrm{GL}_{n-1}\right)$ and $\left(\mathrm{SO}_{n}, \mathrm{SO}_{n-1}\right)$ are strong Gelfand pairs. In a certain sense, these are the only strong Gelfand pairs. In Section 3.2, we gather various characterisations of these pairs and explain, in particular, how coisotropic actions come into play here. For $\mathfrak{s l}_{n}$, it was known for a while that the algebra $\mathcal{C}$ is complete on any regular $G$-orbit, see [KW06, 3.8]. Recently, this completeness result was obtained in the orthogonal case in [CE18]. In both cases, we prove that, for any $x \in \mathfrak{g}$, $\mathrm{C}$ is complete on $G x$ and the $G(n-1)$-action on $G x$ is coisotropic. Moreover, our considerations with nilpotent orbits provide different, simpler proofs in the regular case.

Questions on the completeness of $\mathcal{F}_{a}$ on $G x \subset \mathfrak{g}^{*}$ are related to the Elashvili conjecture, which asserts that ind $\mathfrak{g}^{x}=\mathrm{rk} \mathfrak{g}$ for any $x \in \mathfrak{g}^{*}$. In Section 2, we report on the current state of this conjecture. Theorem 4.3 on the completeness of $\mathcal{C} \subset \mathcal{U}\left(\mathfrak{s l}_{n}\right)$ and the fact that this $\mathcal{C}$ is a limit of MF-subalgebras [Vi91] yield a new proof of Elashvili's conjecture in type A, see Remark 4.5(i). This proof has a potential of being generalised to arbitrary $\mathfrak{g}$. 
Two different geometric features of the Gelfand-Tsetlin construction are discovered in [GS83] and [KW06]. Guillemin and Sternberg in [GS83] work with compact Lie groups over $\mathbb{R}$ and exploit a chain of subalgebras

$$
\mathfrak{u}_{n} \supset \mathfrak{u}_{n-1} \supset \ldots \supset \mathfrak{u}_{1} .
$$

They obtain an integrable system (= complete family of functions), which we call the $\lambda$ system, see Section 4.1 for the relation with the GT-subalgebra $\mathcal{C}$ in type A. Briefly speaking, the $\lambda$-system is generated by the eigenvalues

$$
\left\{\boldsymbol{\lambda}_{k}^{[m]} \mid 1 \leqslant m<n \& 1 \leqslant k \leqslant n-m\right\}
$$

related to the projections $\mathfrak{u}_{n}^{*} \rightarrow \mathfrak{u}_{n-m}^{*}$. This system is examined in details in Section 3.1. The geometric aspect is that it integrates to an action of a compact torus [GS83]. In [KW06], Kostant and Wallach have integrated $\mathcal{C}$ to an action of a unipotent group. We hope to explore related geometric properties of MF-subalgebras in a forthcoming article.

In Section 5, we study actions of reductive subgroups $H \subset G$ on $G x \subset \mathfrak{g}^{*}$. These $H$ actions are obviously Hamiltonian and we show that several numerical characteristics of them, such as defect and corank, are constant along a $G$-sheet $S \subset \mathfrak{g} \simeq \mathfrak{g}^{*}$. This is very much in the spirit of the useful result that the complexity and rank of a $G$-orbit are constant along any sheet $S \subset \mathfrak{g}$, see [P94, Sect. 5]. Building on the insights of [AP14], we prove that the corank does not increase on the closure of a sheet, see Theorem 5.4. Our completeness result for $\mathcal{C}$ in the orthogonal case, arises as an application of this general theory to the pair $(G, H)=\left(\mathrm{SO}_{n}, \mathrm{SO}_{n-1}\right)$.

\section{POISSON BRACKETS AND MishCHENKO-FOMENKO SUbALGEBRAS}

Let $Q$ be a connected affine algebraic group with Lie algebra $\mathfrak{q}$. The symmetric algebra $\mathcal{S}(\mathfrak{q})$ over $\mathbb{k}$ is identified with the graded algebra of polynomial functions on $\mathfrak{q}^{*}$ and we also write $\mathbb{k}\left[\mathfrak{q}^{*}\right]$ for it.

Let $\mathfrak{q}^{\xi}$ denote the stabiliser in $\mathfrak{q}$ of $\xi \in \mathfrak{q}^{*}$. The index of $\mathfrak{q}$, ind $\mathfrak{q}$, is the minimal codimension of $Q$-orbits in $\mathfrak{q}^{*}$. Equivalently, ind $\mathfrak{q}=\min _{\xi \in \mathfrak{q}^{*}} \operatorname{dim} \mathfrak{q}^{\xi}$. By Rosenlicht's theorem [VP89, 2.3], one also has ind $\mathfrak{q}=\operatorname{tr} \cdot \operatorname{deg} \mathbb{k}\left(\mathfrak{q}^{*}\right)^{Q}$. The "magic number" associated with $\mathfrak{q}$ is $\boldsymbol{b}(\mathfrak{q})=(\operatorname{dim} \mathfrak{q}+\operatorname{ind} \mathfrak{q}) / 2$. Since the coadjoint orbits are even-dimensional, the magic number is an integer. If $\mathfrak{q}$ is reductive, then ind $\mathfrak{q}=r \mathfrak{r} \mathfrak{q}$ and $\boldsymbol{b}(\mathfrak{q})$ equals the dimension of a Borel subalgebra. The Poisson-Lie bracket on $\mathbb{k}\left[\mathfrak{q}^{*}\right]$ is defined on the elements of degree 1 (i.e., on $\mathfrak{q}$ ) by $\{x, y\}:=[x, y]$. The Poisson centre of $\mathcal{S}(\mathfrak{q})$ is

$$
\mathcal{S}(\mathfrak{q})^{\mathfrak{q}}=\{H \in \mathcal{S}(\mathfrak{q}) \mid\{H, x\}=0 \quad \forall x \in \mathfrak{q}\} .
$$

Since $Q$ is connected, we also have $\mathcal{S}(\mathfrak{q})^{\mathfrak{q}}=\mathcal{S}(\mathfrak{q})^{Q}=\mathbb{k}\left[\mathfrak{q}^{*}\right]^{Q}$. The set of $Q$-regular elements of $\mathfrak{q}^{*}$ is $\mathfrak{q}_{\text {reg }}^{*}=\left\{\eta \in \mathfrak{q}^{*} \mid \operatorname{dim} \mathfrak{q}^{\eta}=\operatorname{ind} \mathfrak{q}\right\}$. Set $\mathfrak{q}_{\text {sing }}^{*}=\mathfrak{q}^{*} \backslash \mathfrak{q}_{\text {reg }}^{*}$. 
Take $\gamma \in \mathfrak{q}^{*}$. Note that $T_{\gamma}^{*} \mathfrak{q}^{*} \simeq \mathfrak{q}$. Therefore the differential $d_{\gamma} F$ of $F \in \mathcal{S}(\mathfrak{q})$ can be regarded as an element of $\mathfrak{q}$. Let $\hat{\gamma}=\gamma([]$,$) be the skew-symmetric form on \mathfrak{q}$ defined by $\gamma$. In these terms

$$
\left\{F_{1}, F_{2}\right\}(\gamma)=\gamma\left(\left[d_{\gamma} F_{1}, d_{\gamma} F_{2}\right]\right)=\hat{\gamma}\left(d_{\gamma} F_{1}, d_{\gamma} F_{2}\right)
$$

for all $F_{1}, F_{2} \in \mathcal{S}(\mathfrak{q})$. For a subalgebra $\mathcal{A} \subset \mathcal{S}(\mathfrak{q})$, set $d_{\gamma} \mathcal{A}=\left\langle d_{\gamma} F \mid F \in \mathcal{A}\right\rangle_{\mathbb{k}}$. Suppose that $\mathcal{A}$ is Poisson-commutative, i.e., $\{\mathcal{A}, \mathcal{A}\}=0$. Then $\hat{\gamma}$ vanishes on $d_{\gamma} \mathcal{A}$ for each $\gamma \in \mathfrak{q}^{*}$. Clearly $\operatorname{ker} \hat{\gamma}=\mathfrak{q}^{\gamma}$. Hence $\operatorname{dim} d_{\gamma} \mathcal{A} \leqslant \operatorname{dim} \mathfrak{q}^{\gamma}+\frac{1}{2} \operatorname{dim}(Q \gamma)$ and

$$
\operatorname{tr} . \operatorname{deg} \mathcal{A} \leqslant \boldsymbol{b}(\mathfrak{q})
$$

Poisson-commutative subalgebras $\mathcal{A}$ with $\operatorname{tr} \operatorname{deg} \mathcal{A}=\boldsymbol{b}(\mathfrak{q})$ are of particular importance.

Let $\psi_{\gamma}: T_{\gamma}^{*} \mathfrak{q}^{*} \rightarrow T_{\gamma}^{*}(Q \gamma)$ be the canonical projection. Then ker $\psi_{\gamma}=\mathfrak{q}^{\gamma}$. The skewsymmetric form $\hat{\gamma}$ is non-degenerate on $T_{\gamma}^{*}(Q \gamma)$. The algebra $\mathbb{k}[Q \gamma]$ carries the Poisson structure, which is defined by (1.1) with $F_{1}, F_{2} \in \mathbb{k}[Q \gamma]$ and which is inherited from $\mathfrak{q}^{*}$. Once again, $\left\{\left.F_{1}\right|_{Q \gamma},\left.F_{2}\right|_{Q \gamma}\right\}=\left.\left\{F_{1}, F_{2}\right\}\right|_{Q \gamma}$ for all $F_{1}, F_{2} \in \mathcal{S}(\mathfrak{q})$. The coadjoint orbit $Q \gamma$ is a smooth symplectic variety.

Definition 1. A set $\left\{F_{1}, \ldots, F_{m}\right\} \subset \mathbb{k}[Q \gamma]$ is said to be a complete family in involution if $F_{1}, \ldots, F_{m}$ are algebraically independent, $\left\{F_{i}, F_{j}\right\}=0$ for all $i, j$, and $m=\frac{1}{2} \operatorname{dim}(Q \gamma)$.

Let $\mathcal{A} \subset \mathcal{S}(\mathfrak{q})$ be a Poisson-commutative subalgebra. Then the restriction of $\mathcal{A}$ to $Q \gamma$, denoted $\left.\mathcal{A}\right|_{Q \gamma}$, is Poisson-commutative for every $\gamma$. We say that $\mathcal{A}$ is complete on $Q \gamma$, if $\left.\mathcal{A}\right|_{Q \gamma}$ contains a complete family in involution. The condition is equivalent to the equality $\operatorname{tr} . \operatorname{deg}\left(\left.\mathcal{A}\right|_{Q \gamma}\right)=\frac{1}{2} \operatorname{dim}(Q \gamma)$.

Lemma 1.1. Suppose that $\mathcal{A} \subset \mathcal{S}(\mathfrak{q})$ is Poisson-commutative, $\gamma \in \mathfrak{q}_{\text {reg }}^{*}$, and $\operatorname{dim} d_{\gamma} \mathcal{A}=\boldsymbol{b}(\mathfrak{q})$. Then $\mathcal{A}$ is complete on $Q \gamma$.

Proof. Since $\gamma$ is regular, we have $\operatorname{dim} \operatorname{ker} \psi_{\gamma}=$ ind $\mathfrak{q}$. Therefore

$$
\operatorname{dim} \psi_{\gamma}\left(\boldsymbol{d}_{\gamma} \mathcal{A}\right) \geqslant \boldsymbol{b}(\mathfrak{q})-\operatorname{ind} \mathfrak{q}=\frac{1}{2} \operatorname{dim}(Q \gamma)
$$

as required.

The celebrated "argument shift method", which goes back to Mishchenko-Fomenko [MF78], provides a large Poisson-commutative subalgebras of $\mathcal{S}(\mathfrak{q})$ starting from the Poisson centre $\mathcal{S}(\mathfrak{q})^{\mathfrak{q}}$. Given $\gamma \in \mathfrak{q}^{*}$, the $\gamma$-shift of argument produces the Mishchenko-Fomenko subalgebra $\mathcal{F}_{\gamma}$. Namely, for $F \in \mathcal{S}(\mathfrak{q})=\mathbb{k}\left[\mathfrak{q}^{*}\right]$, let $\partial_{\gamma} F$ be the direction derivative of $F$ with respect to $\gamma$, i.e.,

$$
\partial_{\gamma} F(x)=\left.\frac{d}{d t} F(x+t \gamma)\right|_{t=0} .
$$

Then $\mathcal{F}_{\gamma}$ is generated by all $\partial_{\gamma}^{k} F$ with $k \geqslant 0$ and $F \in \mathcal{S}(\mathfrak{q})^{\mathfrak{q}}$. The core of this method is that for any $\gamma \in \mathfrak{q}^{*}$ there is the Poisson bracket $\{,\}_{\gamma}$ on $\mathfrak{q}^{*}$ such that $\{\xi, \eta\}_{\gamma}=\gamma([\xi, \eta])$ for 
$\xi, \eta \in \mathfrak{q}$, and that this new bracket is compatible with $\{$,$\} . Two Poisson brackets on \mathcal{S}(\mathfrak{q})$ are said to be compatible, if all their linear combinations are again Poisson brackets. For more details see [DZ05, Sect. 1.8.3].

1.1. Compatible brackets and pencils of skew-symmetric forms. Take $\gamma \in \mathfrak{q}^{*}$ and let $\mathcal{F}_{\gamma}$ be the corresponding MF-subalgebra of $\mathcal{S}(\mathfrak{q})$. The original description of $\mathcal{F}_{\gamma}$ [MF78] was different from (but equivalent to) the one presented above. For $F \in \mathcal{S}(\mathfrak{q})$ and $t \in \mathbb{k}$, let $F_{\gamma, t}$ be a function on $\mathfrak{q}^{*}$ such that $F_{\gamma, t}(x)=F(x+t \gamma)$ for each $x \in \mathfrak{q}^{*}$. Suppose that $\operatorname{deg} F=m$. Then $F_{\gamma, t}$ expands as a polynomial in $t$ as

$$
F_{\gamma, t}=F^{(0)}+t F^{(1)}+\ldots+t^{m} F^{(m)},
$$

where $F^{(k)}=\frac{1}{k !} \partial_{\gamma}^{k} F$. As we have stated above, $\mathcal{F}_{\gamma}$ is generated by all elements $F^{(k)}$ associated with all $F \in \mathcal{S}(\mathfrak{q})^{\mathfrak{q}}$. A standard argument with the Vandermonde determinant shows that $\mathcal{F}_{\gamma}$ is generated by $F_{\gamma, t}$ with $F \in \mathcal{S}(\mathfrak{q})^{\mathfrak{q}}$ and $t \in \mathbb{k}$. It is also clear that if $\mathcal{S}(\mathfrak{q})^{\mathfrak{q}}$ is generated by $F_{1}, \ldots, F_{n}$, then $\mathcal{F}_{\gamma}$ is generated by $F_{i}^{(k)}$ with $i=1, \ldots, n$ and all $k$.

Consider the map $\varphi_{t}: \mathfrak{q}^{*} \rightarrow \mathfrak{q}^{*}$ such that $\varphi_{t}(x)=x-t \gamma$ for $x \in \mathfrak{q}^{*}$. It extends in the usual way to $\mathbb{k}\left[\mathfrak{q}^{*}\right]$ and then $F_{\gamma, t}=\varphi_{t}(F)$. The map $\varphi_{t}$ defines a new Poisson bracket on $\mathfrak{q}^{*}$ by the formula

$$
\left\{F_{1}, F_{2}\right\}_{t}(x)=\left\{\varphi_{t}\left(F_{1}\right), \varphi_{t}\left(F_{2}\right)\right\}\left(\varphi_{t}(x)\right),
$$

where $F_{1}, F_{2} \in \mathbb{k}\left[\mathfrak{q}^{*}\right]$. For $\xi, \eta \in \mathfrak{q}$, the formula reeds

$$
\{\xi, \eta\}_{t}=\varphi_{t}^{-1}\left(\left\{\varphi_{t}(\xi), \varphi_{t}(\eta)\right\}\right)=[\xi, \eta]-t \hat{\gamma}(\xi, \eta) .
$$

The Poisson algebras $(\mathcal{S}(\mathfrak{q}),\{\}$,$) and \left(\mathcal{S}(\mathfrak{q}),\{,\}_{t}\right)$ are isomorphic. The MF-subalgebra $\mathcal{F}_{\gamma}$ is generated by $\varphi_{t}^{-1}\left(\mathcal{S}(\mathfrak{g})^{\mathfrak{g}}\right)(t \in \mathbb{k})$, i.e., by the Poisson centres of $\left(\mathcal{S}(\mathfrak{q}),\{,\}_{t}\right)$ with $t \in \mathbb{k}$. For $F \in \mathcal{S}(\mathfrak{g})^{\mathfrak{g}}$, we have

$$
\left\{F_{\gamma, t}, F_{\gamma, s}\right\}_{t}=0=\left\{F_{\gamma, t}, F_{\gamma, s}\right\}_{s}
$$

and therefore $\left\{F_{\gamma, t}, F_{\gamma, s}\right\}=0$ if $t \neq s$. Using the continuity, one concludes that $\mathcal{F}_{\gamma}$ is Poisson-commutative.

Suppose that we wish to calculate $\operatorname{dim} d_{x} \mathcal{F}_{\gamma}$. The differential $d_{x} F_{\gamma, t}=d_{x+t \gamma} F$ lies in the kernel of the skew-symmetric form $\hat{x}_{t}=\hat{x}+t \hat{\gamma}$ if $F \in \mathcal{S}(\mathfrak{q})^{\mathfrak{q}}$. Therefore

$$
d_{x} \mathcal{F}_{\gamma} \subset \sum_{t \in \mathbb{k}} \operatorname{ker}(\hat{x}+t \hat{\gamma}) .
$$

We consider below the following conditions on $\mathfrak{q}, x, \gamma$ :

$$
\left\{\begin{array}{l}
(1) \quad \operatorname{tr} \cdot \operatorname{deg} \mathcal{S}(\mathfrak{q})^{\mathfrak{q}}=\operatorname{ind} \mathfrak{q}, \\
(2) \quad(x+\mathbb{k} \gamma) \cap \mathfrak{q}_{\text {reg }}^{*} \neq \varnothing \\
(3) \quad \text { there is at least one } \lambda \in \mathbb{k} \text { such that } d_{y}\left(\mathcal{S}(\mathfrak{q})^{\mathfrak{q}}\right)=\mathfrak{q}^{y} \text { for } y=x+\lambda \gamma
\end{array}\right.
$$


Note that (3) implies (1) and (2). There are tricks that allow one to lift (1), but we are not going to consider them. Condition (2) is quite harmless, it is satisfied if $\gamma \in \mathfrak{q}_{\text {reg }}^{*}$ or $x \in \mathfrak{q}_{\text {reg }}^{*}$.

Lemma 1.2. Suppose that (3) of (1.5) holds. Then

$$
d_{x} \mathcal{F}_{\gamma}=\sum_{x+t \gamma \in \mathfrak{q}_{\mathrm{reg}}^{*}} \operatorname{ker}(\hat{x}+t \hat{\gamma})=: L(x, \gamma)
$$

Proof. Condition (3) implies that there is a non-empty open subset $Y \subset(x+\mathbb{k} \gamma)$ such that $d_{y}\left(\mathcal{S}(\mathfrak{q})^{\mathfrak{q}}\right)=\mathfrak{q}^{y}=\operatorname{ker} \hat{y}$ for all $y \in Y$. Thus, $\sum_{y \in Y} \mathfrak{q}^{y} \subset d_{x} \mathcal{F}_{\gamma}$.

For almost all $t \in \mathbb{k}$, we have $x+t \gamma \in \mathfrak{q}_{\text {reg }}^{*}$. If $x^{\prime}=x+t_{0} \gamma \in \mathfrak{q}_{\text {sing }}^{*}$, then nevertheless $d_{x^{\prime}} F_{\gamma, t_{0}}=\lim _{t \rightarrow t_{0}} d_{x} F_{\gamma, t}$, where we can assume that $x+t \gamma \in \mathfrak{q}_{\text {reg }}^{*}$. Here $d_{x} F_{\gamma, t} \in L(x, \gamma)$ and hence $d_{x^{\prime}} F_{\gamma, t_{0}} \in L(x, \gamma)$ as well. Now we have

$$
\sum_{y \in Y} \mathfrak{q}^{y} \subset d_{x} \mathcal{F}_{\gamma} \subset L(x, \gamma)
$$

According to [PY08, Lemma A.1], $\sum_{y \in Y} \mathfrak{q}^{y}=L(x, \gamma)$. This concludes the proof.

Assume also that $\hat{x}$ and $\hat{\gamma}$ are not proportional. Now the problem is to deal with the pencil of skew-symmetric forms on $\mathfrak{q}$ generated by $\hat{x}$ and $\hat{\gamma}$.

Let $\mathcal{P}$ be a two-dimensional vector space of (possibly degenerate) skew-symmetric bilinear forms on a finite-dimensional vector space $V$. Set $m=\max _{A \in \mathcal{P}}$ rk $A$, and let $\mathcal{P}_{\text {reg }} \subset \mathcal{P}$ be the set of all forms of rank $m$. Then $\mathcal{P}_{\text {reg }}$ is a conical open subset of $\mathcal{P}$. For each $A \in \mathcal{P}$, let ker $A \subset V$ be the kernel of $A$. Our object of interest is the subspace $L:=\sum_{A \in \mathcal{P}_{\text {reg }}} \operatorname{ker} A$.

Proposition 1.3 (see [T91, Thm 1(d)]). Take non-proportional $A, B \in \mathcal{P}_{\text {reg. Then there is the }}$ so-called Jordan-Kronecker canonical form of $A$ and $B$. Namely, $V=V_{1} \oplus \ldots \oplus V_{d}$, where $A\left(V_{i}, V_{j}\right)=0=B\left(V_{i}, V_{j}\right)$ for $i \neq j$, and accordingly, $A=\sum A_{i}$ and $B=\sum B_{i}$. There are two possibilities for $\left(A_{i}, B_{i}\right)$, one obtains either a Kronecker or a Jordan block here, see figures below. Assume that $\operatorname{dim} V_{i}>0$ for each $i$. 
$A_{i}$

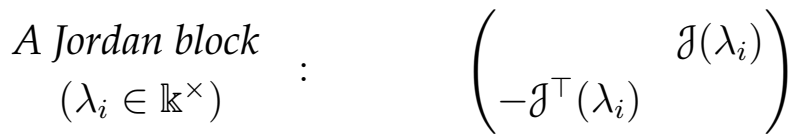

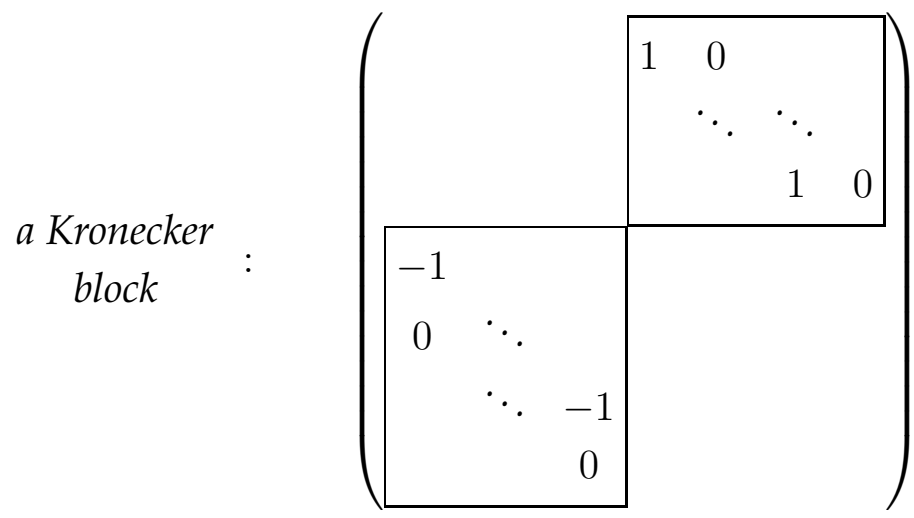

$B_{i}$

$\left(\begin{array}{ll} & -\mathrm{I} \\ \mathrm{I} & \end{array}\right)$

where $\mathcal{J}\left(\lambda_{i}\right)=\left(\begin{array}{cccc}\lambda_{i} & 1 & & \\ & \lambda_{i} & \ddots & \\ & & \ddots & 1 \\ & & & \lambda_{i}\end{array}\right)$.

Remark. In general, there can occur "Jordan blocks with $\lambda_{i}=\infty$ ", but this is not the case here, since $B \in \mathcal{P}_{\text {reg. }}$. Since $A \in \mathcal{P}_{\text {reg }}$ as well, the case of $\lambda_{i}=0$ doesn't occur either.

Proposition 1.4. (i) For each non-zero $C \in \mathcal{P}$, we have $\operatorname{dim}(L \cap \operatorname{ker} C)=\operatorname{dim} V-m$.

(ii) If $\mathcal{P}_{\text {reg }}=\mathcal{P} \backslash\{0\}$, then $\operatorname{dim} L=\operatorname{dim} V-\frac{m}{2}$.

(iii) Suppose that $C \in \mathcal{P}, C \neq 0$, and $C \notin \mathcal{P}_{\text {reg. }}$. Then $\operatorname{dim} L \leqslant(\operatorname{dim} V-m)+\frac{1}{2} \mathrm{rk} C$ and $\operatorname{dim} L=(\operatorname{dim} V-m)+\frac{1}{2} \mathrm{rk} C$ if and only if $\mathcal{P} \backslash \mathcal{P}_{\mathrm{reg}}=\mathbb{k} C, \mathrm{rk}\left(\left.A\right|_{\operatorname{ker} C}\right)=\operatorname{dim} \operatorname{ker} C-\operatorname{dim} V+m$ for $A \in \mathcal{P}_{\text {reg. }}$.

Proof. We choose non-proportional $A, B \in \mathcal{P}_{\text {reg }}$ and bring them into a Jordan-Kronecker form according to Proposition 1.3. Keep the above notation. In particular, $V=V_{1} \oplus \ldots \oplus V_{d}$. For any $C \in \mathcal{P}$, we have $C=\sum C_{i}$ accordingly.

Note that if $V_{i}$ gives rise to a Jordan block, then $\operatorname{dim} V_{i}$ is even and both $A_{i}$ and $B_{i}$ are non-degenerate on $V_{i}$. For a Kronecker block, $\operatorname{dim} V_{i}=2 k_{i}+1$, rk $A_{i}=2 k_{i}=\mathrm{rk} B_{i}$ and the same holds for every non-zero linear combination of $A_{i}$ and $B_{i}$. 
Let us assume that $V_{i}$ defines a Kronecker block if and only if $1 \leqslant i \leqslant d^{\prime}$. Then necessarily $d^{\prime}=\operatorname{dim} V-m$. We have

$$
L=\bigoplus_{i=1}^{d^{\prime}} \sum_{C \in \mathcal{P}_{\mathrm{reg}}} \operatorname{ker} C_{i}=: \bigoplus_{i=1}^{d^{\prime}} L_{i} .
$$

It follows from the matrix form of a Kronecker block that $L_{i}$ is the linear span of the last $(k+1)$ vectors in the basis of $V_{i}$. Hence $\operatorname{dim} L_{i}=k_{i}+1$. For any non-zero $C \in \mathcal{P}$, we have $\operatorname{ker} C \cap L=\bigoplus_{i=1}^{d^{\prime}}\left(\operatorname{ker} C \cap L_{i}\right)$, also $\operatorname{dim} \operatorname{ker} C_{i}=1$ and $\operatorname{ker} C_{i} \subset L_{i}$ for each $i \leqslant d^{\prime}$. Thereby $\operatorname{dim}(\operatorname{ker} C \cap L)=d^{\prime}$. Thus, (i) is settled.

If $\lambda=\lambda_{i}$ for $\lambda_{i}$ coming from a Jordan block, then $C=A+\lambda B \notin \mathcal{P}_{\text {reg }}$ and $C \neq 0$. Hence the equality $\mathcal{P} \backslash\{0\}=\mathcal{P}_{\text {reg }}$ takes place if and only if there are no Jordan blocks. In this case $\operatorname{dim} L=(\operatorname{dim} V+d) / 2$. Part (ii) is settled as well.

(iii) By the assumptions on $C$, up to a non-zero scalar factor $C=A+\lambda_{i} B$, where $\lambda_{i}$ comes from a Jordan block. We have $\operatorname{dim} L=d^{\prime}+\frac{1}{2} \sum_{j=1}^{d^{\prime}} \mathrm{rk} C_{j}$. Clearly $\sum_{j=1}^{d^{\prime}} \mathrm{rk} C_{j} \leqslant \mathrm{rk} C$. The equality takes place if and only if $C_{j}=0$ for $j>d^{\prime}$. Further, $C_{j}=0$ if and only if $\lambda_{j}=\lambda_{i}$ and $\operatorname{dim} V_{j}=2$. The first condition, $\lambda_{i}=\lambda_{j}$, is satisfied if and only if $\mathcal{P} \backslash \mathcal{P}_{\text {reg }}=\mathbb{k} C$. Until the end of the proof assume that $\lambda_{i}=\lambda_{j}$ for all $j>d^{\prime}$.

Set $U=\operatorname{ker} C$. Note that $A$ and $C$ generate $\mathcal{P}$. Therefore rk $\left(\left.A^{\prime}\right|_{U}\right)=\operatorname{rk}\left(\left.A\right|_{U}\right)$ for every $A^{\prime} \in \mathcal{P}_{\text {reg. }}$. Recall that $\operatorname{dim} \operatorname{ker} C_{j}=1$ if $j \leqslant d^{\prime}$. Since $U=\bigoplus_{j=1}^{d} \operatorname{ker} C_{j}$ and the spaces $\left\{\operatorname{ker} C_{j}\right\}$ are pairwise orthogonal w.r.t. any form in $\mathcal{P}$, we have $A\left(\operatorname{ker} C_{j}, U\right)=0$ for $j \leqslant d^{\prime}$. Hence the condition rk $\left(\left.A\right|_{U}\right)=\operatorname{dim} U-\operatorname{dim} V+m$ implies that $A_{j}$ is non-degenerate on $\operatorname{ker} C_{j}$ for any $j>d^{\prime}$. The explicit matrix form of a Jordan block shows that $\operatorname{ker} C_{j}$ is spanned by two middle basis vectors of $V_{j}$. Therefore, $A_{j}$ is non-degenerate on $\operatorname{ker} C_{j}$ if and only if $\operatorname{dim} V_{j}=2$. This completes the proof.

Corollary 1.5. Suppose that (3) of (1.5) holds for $x$ and $\gamma$. Then $\operatorname{dim}\left(d_{x} \mathcal{F}_{\gamma} \cap \mathfrak{q}^{x}\right)=$ ind $\mathfrak{q}$ and $\operatorname{dim} d_{x} \mathcal{F}_{\gamma} \leqslant \operatorname{ind} \mathfrak{q}+\frac{1}{2} \operatorname{dim}(Q x)$. Assume additionally that $\hat{x}$ and $\hat{\gamma}$ are non-proportional. Then

$$
\operatorname{dim} d_{x} \mathcal{F}_{\gamma}=\operatorname{ind} \mathfrak{q}+\frac{1}{2} \operatorname{dim}(Q x)
$$

if and only if $(\mathbb{k} x \oplus \mathbb{k} \gamma) \cap \mathfrak{q}_{\text {sing }}^{*} \subset \mathbb{k} x$ and $\operatorname{dim}\left(\mathfrak{q}^{x}\right)^{\bar{\gamma}}=$ ind $\mathfrak{q}$ for the restriction $\bar{\gamma}=\left.\gamma\right|_{\mathfrak{q}^{x}}$.

Proof. Consider first the case, where $\operatorname{dim}(\mathbb{k} \hat{x}+\mathbb{k} \hat{\gamma}) \leqslant 1$. Suppose that (3) holds for $y \in x+t \gamma$. Then $d_{x} \mathcal{F}_{\gamma}=d_{y}\left(\mathcal{S}(\mathfrak{q})^{\mathfrak{q}}\right)=\mathfrak{q}^{y}$. Here $y$ is necessary regular and $\operatorname{dim} \mathfrak{q}^{y}=$ ind $\mathfrak{q}$.

Suppose now that $\hat{x}$ and $\hat{\gamma}$ are non-proportional. By Lemma 1.2, $d_{x} \mathcal{F}_{\gamma}=L(x, \gamma)$, where $L(x, \gamma)=\sum_{\hat{y} \in \mathcal{P}_{\text {reg }}} \operatorname{ker} \hat{y}$ for $\mathcal{P}=\mathbb{k} \hat{x} \oplus \mathbb{k} \hat{\gamma}$. According to Proposition 1.4, we have

$$
\operatorname{dim}(\operatorname{ker} \hat{x} \cap L(x, \gamma))=\operatorname{ind} \mathfrak{q} \text { and } \operatorname{dim} L(x, \gamma) \leqslant \operatorname{ind} \mathfrak{q}+\frac{1}{2} \operatorname{dim}(Q x) .
$$


By the same proposition, the inequality turns into equality if and only if $\mathcal{P} \backslash \mathcal{P}_{\text {reg }} \subset \mathbb{k} \hat{x}$ and $\operatorname{dim}\left(\mathfrak{q}^{x}\right)^{\bar{\gamma}}=$ ind $\mathfrak{q}$ in case $x \in \mathfrak{q}_{\text {sing. }}^{*}$. Note that $\mathfrak{q}^{y}$ is Abelian for any $y \in \mathfrak{q}_{\text {reg }}^{*}$, see e.g. [P03, Sect. 1], and therefore ind $\mathfrak{q}^{y}=\operatorname{dim} \mathfrak{q}^{y}=\operatorname{ind} \mathfrak{q},\left(\mathfrak{q}^{y}\right)_{\text {reg }}^{*}=\left(\mathfrak{q}^{y}\right)^{*}$ in this case.

Remark 1.6. (i) An idea how to estimate tr.deg $\left(\left.\mathcal{F}_{\gamma}\right|_{Q x}\right)$ appeared in [B91], see also [BZ16], especially for the use of Jordan-Kronecker blocks.

(ii) The Poisson-commutativity of $\mathcal{F}_{\gamma}$ can be shown using pencils of skew-symmetric forms. The equality $\left\{\mathcal{F}_{\gamma}, \mathcal{F}_{\gamma}\right\}=0$ holds if and only if $\hat{x}\left(d_{x} \mathcal{F}_{\gamma}, d_{x} \mathcal{F}_{\gamma}\right)=0$ for generic $x \in \mathfrak{q}^{*}$. In case $\gamma=0$, we have $\mathcal{F}_{0}=\mathcal{S}(\mathfrak{q})^{\mathfrak{q}}$ and there is nothing to prove. Suppose that $x \in \mathfrak{q}_{\text {reg }}^{*}$ and that $\hat{\gamma}$ and $\hat{x}$ are non-proportional. By the same continuity principle, which has been used in the proof of Lemma 1.2, $d_{x} \mathcal{F}_{\gamma} \subset L(x, \gamma)$. Suppose that $\xi \in \operatorname{ker}(\hat{x}+\lambda \hat{\gamma}) \subset L(x, \gamma)$. Making use of [PY08, Lemma A.1], one writes

$$
L(x, \gamma)=\sum_{x+t \gamma q_{\mathrm{reg}}^{*}, t \neq \lambda} \operatorname{ker}(\hat{x}+t \hat{\gamma}) .
$$

Let $\eta \in \operatorname{ker}(\hat{x}+\mu \hat{\gamma}) \subset L(x, \gamma)$ with $\mu \neq \lambda$. Then

$$
(\mu-\lambda) \hat{x}(\xi, \eta)=\mu(\hat{x}+\lambda \hat{\gamma})(\xi, \eta)-\lambda(\hat{x}+\mu \hat{\gamma})(\xi, \eta)=0+0=0 .
$$

Thus, $\hat{x}(\xi, \eta)=0$ and $\hat{x}$ vanishes on $d_{x} \mathcal{F}_{\gamma}$.

\section{COMPlete SUbALGEBRAS AND NILPOTENT ORBITS}

In this section, $G$ is a connected reductive $\mathbb{k}$-group and $\mathfrak{g}=\operatorname{Lie} G$. Set $l=\operatorname{ind} \mathfrak{g}=\mathrm{rk} \mathfrak{g}$. By a classical result of Chevalley, $\mathcal{S}(\mathfrak{g})^{\mathfrak{g}}=\mathbb{k}\left[H_{1}, \ldots, H_{l}\right]$, where the $H_{i}$ 's are homogeneous and algebraically independent. Furthermore, $\sum_{j=1}^{l} \operatorname{deg} H_{j}=\boldsymbol{b}(\mathfrak{g})$. Take $a \in \mathfrak{g}^{*}$. Recall that the MF-subalgebra $\mathcal{F}_{a} \subset \mathcal{S}(\mathfrak{g})$ is generated by the direction derivatives $\partial_{a}^{k} H_{i}$ with $1 \leqslant i \leqslant l$ and $0 \leqslant k \leqslant \operatorname{deg} H_{i}-1$.

Fix an isomorphism $\mathfrak{g}^{*} \simeq \mathfrak{g}$ of $G$-modules. Making use of this isomorphism, we transfer the standard terminology for $\mathfrak{g}$ to the elements of $\mathfrak{g}^{*}$, e.g. while referring to nilpotent and semisimple elements of $\mathfrak{g}^{*}$, considering sheets, etc.

Our main concern in this section is the following question:

$$
\text { Is } \mathcal{F}_{a} \text { complete on an orbit } G x \subset \mathfrak{g}^{*} \text { ? }
$$

For $G x=\{x\}$, any choice of $a$ leads to a complete subalgebra. Therefore we consider only $G x$ with $\operatorname{dim}(G x) \geqslant 2$. It is reasonable to assume that $a \in \mathfrak{g}_{\mathrm{reg}}^{*}$. Whenever computing $\operatorname{dim} d_{x} \mathcal{F}_{a}$ we will suppose that $\hat{a}$ and $\hat{x}$ are non-proportional. This can be achieved by taking some other $x^{\prime} \in G x$ instead of $x$.

Lemma 2.1. Take $a \in \mathfrak{g}_{\text {reg }}^{*}$. Then $\operatorname{dim}\left(d_{x} \mathcal{F}_{a} \cap \mathfrak{g}^{x}\right)=l$ for each $x \in \mathfrak{g}^{*}$. Furthermore, $\mathcal{F}_{a}$ is complete on $G y \neq\{y\}$ if and only if ind $\mathfrak{g}^{y}=l$ and there is $x \in G y$ such that

(i) $(\mathbb{k} x \oplus \mathbb{k} a) \cap \mathfrak{g}_{\text {sing }}^{*} \subset \mathbb{k} x$, 
(ii) $\bar{a} \in\left(\mathfrak{g}^{x}\right)_{\mathrm{reg}}^{*}$ for the restriction $\bar{a}=\left.a\right|_{\mathfrak{g}^{x}}$.

Proof. First, let us examine the conditions in (1.5). Clearly, $\operatorname{tr} \cdot \operatorname{deg} \mathcal{S}(\mathfrak{g})^{\mathfrak{g}}=$ ind $\mathfrak{g}$. Since $a$ is regular, (2) holds as well. By the Kostant regularity criterion [K63, Thm 9],

$$
\left\langle d_{\xi} H_{j} \mid 1 \leqslant j \leqslant l\right\rangle_{\mathbb{k}}=\mathfrak{g}^{\xi} \text { if and only if } \xi \in \mathfrak{g}_{\text {reg }}^{*} .
$$

Hence (2) implies (3). Now we are ready to use Corollary 1.5. It asserts, in particular, that $\operatorname{dim}\left(\mathcal{F}_{a} \cap \mathfrak{g}^{x}\right)=l$ for each $x \in \mathfrak{g}^{*}$. In view of this, $\mathcal{F}_{a}$ is complete on $G y$ if and only if there is $x \in G y$ such that $\operatorname{dim} d_{x} \mathcal{F}_{a}=l+\frac{1}{2} \operatorname{dim}(G x)$. W.l.o.g. assume that $\hat{x}$ and $\hat{a}$ are non-proportional. Then by Corollary 1.5, the equality $\operatorname{dim} d_{x} \mathcal{F}_{a}=l+\frac{1}{2} \operatorname{dim}(G x)$ takes place if and only if $(\mathbb{k} x \oplus \mathbb{k} a) \cap \mathfrak{g}_{\text {sing }}^{*} \subset \mathbb{k} x$ and $\operatorname{dim}\left(\mathfrak{g}^{x}\right)^{\bar{a}}=l$.

Consider the condition $\operatorname{dim}\left(\mathfrak{g}^{x}\right)^{\bar{a}}=l$. It implies that ind $\mathfrak{g}^{x} \leqslant l$. At the same time ind $\mathfrak{g}^{x} \geqslant$ ind $\mathfrak{g}$ by Vinberg's inequality, see [P03, Cor. 1.7]. If this condition is satisfied, then ind $\mathfrak{g}^{x}=l$. In the other direction, if ind $\mathfrak{g}^{x}=l$, then $\operatorname{dim}\left(\mathfrak{g}^{x}\right)^{\bar{a}}=l$ if and only if $\bar{a} \in\left(\mathfrak{g}^{x}\right)_{\text {reg }}^{*}$.

Corollary 2.2. Keep the assumption $a \in \mathfrak{g}_{\text {reg }}^{*}$. Then $\mathcal{F}_{a}$ is complete on $G y$ if and only if there is $x \in$ Gy such that $\operatorname{dim} d_{x} \mathcal{F}_{a}=l+\frac{1}{2} \operatorname{dim}(G x)$.

The assertion

$$
\text { ind } \mathfrak{g}^{x}=\mathrm{rk} \mathfrak{g} \text { for each } x \in \mathfrak{g}
$$

is known as Elashvili's conjecture. It has no fully conceptual proof in spite of many efforts. However, the equality obviously holds for all regular and all semisimple elements. Elashvili's conjecture is proven for the classical Lie algebras [Y06] and for all Richardson elements [CM10]. It is also checked for the exceptional $\mathfrak{g}$ [dG08, CM10]. We take it for granted that Elashvili's conjecture is true. Therefore, for any orbit $G x \subset \mathfrak{g}^{*}$, there is an element $a \in \mathfrak{g}_{\text {reg }}^{*}$ such that the MF-subalgebra $\mathcal{F}_{a}$ is complete on $G x$, see [B91] and also [MY17, Sect. 2].

Return for a while to an arbitrary algebraic Lie algebra $\mathfrak{q}=\operatorname{Lie} Q$. Take $a, x \in \mathfrak{q}^{*}$ and let $F \in \mathcal{S}(\mathfrak{q})$ be a homogeneous polynomial of degree $d$. Then

$$
(d-k-1) ! d_{x}\left(\partial_{a}^{k} F\right)=(k-1) ! d_{a}\left(\partial_{x}^{d-k-1} F\right)
$$

and therefore

$$
d_{x} \mathcal{F}_{a}=d_{a} \mathcal{F}_{x}
$$

as a subspace of $\mathfrak{q}$.

Theorem 2.3. Suppose that $a, x \in \mathfrak{q}_{\text {reg }}^{*}$ and that $\mathfrak{q}, \gamma=a$, and $x$ satisfy (1.5). Then $\mathcal{F}_{a}$ is complete on $Q x$ if and only if $\mathcal{F}_{x}$ is complete on $Q a$. 
Proof. Clearly (1.5) holds for $a$ and generic points $x^{\prime} \in Q x$. Suppose that $\mathcal{F}_{a}$ is complete on $Q x$. By Lemma 1.1 and Corollary 1.5, this is the case if and only if there is $q \in Q$ such that $\operatorname{dim} d_{q x} \mathcal{F}_{a}=\boldsymbol{b}(\mathfrak{q})$. As one can easily see, $q d_{x} \mathcal{F}_{a}=d_{q x} \mathcal{F}_{q a}$. Combining this $Q-$ equivariance with (2.3), we conclude that $\operatorname{dim} d_{q^{-1} a} \mathcal{F}_{x}=\operatorname{dim} d_{q x} \mathcal{F}_{a}=\boldsymbol{b}(\mathfrak{q})$. The equality $\operatorname{dim} d_{q^{-1} a} \mathcal{F}_{x}=\boldsymbol{b}(\mathfrak{q})$ implies that $\mathcal{F}_{x}$ is complete on $Q a$, see Lemma 1.1.

2.1. By a result of Tarasov [T02], if $a \in \mathfrak{g}_{\text {reg }}^{*}$ is semisimple, then $\mathcal{F}_{a}$ is complete on every coadjoint orbit $G x \subset \mathfrak{g}_{\text {reg }}^{*}$. See also [K09] for its applications. As the next step, we lift the assumption that $a$ is semisimple and also allow $x$ to be regular or semisimple.

Theorem 2.4. Let $a \in \mathfrak{g}_{\text {reg. }}^{*}$. The MF-subalgebra $\mathcal{F}_{a}$ is complete on $G x$ whenever $x$ is semisimple or regular. In other words, $\mathcal{F}_{a}$ is complete on each closed or regular (co)adjoint orbit.

Proof. Let $\{e, h, f\}$ be a principal $\mathfrak{s l}_{2}$-triple in $\mathfrak{g}$ and $\mathfrak{b}=$ Lie $B$ be the unique Borel subalgebra that contains $e$. Then $\mathfrak{g}^{e} \subset \mathfrak{b}$ and $\mathcal{K}=f+\mathfrak{g}^{e}$ is the associated Kostant section in $\mathfrak{g} \simeq \mathfrak{g}^{*}$. By [K63], $G \mathcal{K}=\mathfrak{g}_{\text {reg }}^{*}$. Clearly $G \mathfrak{b}=\mathfrak{g}$. W.l.o.g. we may assume that $a=f+y \in \mathcal{K}$. Take $x \in \mathfrak{b}$.

Suppose that $x$ is semisimple or regular. In the first case, $\mathfrak{g}^{x}$ is reductive and clearly ind $\mathfrak{g}^{x}=\mathrm{rk} \mathfrak{g}^{x}=\mathrm{rk} \mathfrak{g}$. In the second, $\operatorname{dim} \mathfrak{g}^{x}=l=\operatorname{ind} \mathfrak{g}^{x}$. Now it suffices to verify conditions (i) and (ii) of Lemma 2.1 for the pair $(a, x)$. Note that (ii) holds for each $a \in \mathfrak{g}^{*}$ if $x$ is regular.

(i) A generic element of the plane $\langle a, x\rangle_{\mathrm{k}}$ is of the form $\alpha(f+y)+\beta x=\alpha f+(\alpha y+\beta x)$, where $y, x \in \mathfrak{b}$. If $\alpha \neq 0$, then all these elements are regular in $\mathfrak{g}^{*}$, in view of a classical result of Kostant. Indeed, he proved that $f+\mathfrak{b} \subset \mathfrak{g}_{\text {reg }}$, see [K63].

(ii) Under the assumption that $x$ is semisimple, we have $x \in B \mathfrak{t}$, where $\mathfrak{t}=\mathfrak{g}^{h} \subset \mathfrak{b}$ is a Cartan subalgebra. W.l.o.g. assume that $x \in \mathfrak{t}$. Then $\mathfrak{g}^{x}=\mathfrak{l}$ is a standard Levi subalgebra. Further, $\bar{f}=\left.f\right|_{\mathfrak{r}}$ is a regular nilpotent element of $\mathfrak{l}$ and it can be included into a principal $\mathfrak{s l}_{2}$-triple $\{\tilde{e}, \tilde{h}, \bar{f}\} \subset \mathfrak{l}$ such that $\tilde{h} \in \mathfrak{t}$. Note that $\mathfrak{l} \cap \mathfrak{b}$ is the unique Borel subalgebra of $\mathfrak{l}$ containing $\tilde{e}$. We have $\bar{a}=\bar{f}+\bar{y} \in \mathfrak{l}^{*} \simeq \mathfrak{l}$, where $\bar{y} \in \mathfrak{l} \cap \mathfrak{b}$. By the same result of Kostant $[\mathrm{K} 63], \bar{f}+(\mathfrak{l} \cap \mathfrak{b}) \subset \mathfrak{l}_{\text {reg }}$, and therefore $\bar{a} \in \mathfrak{l}_{\text {reg }}^{*}$.

One is tempted to generalise Theorem 2.4 to all elements $x \in \mathfrak{b}$. The obstacle is that finding a regular $a \in \mathfrak{g}^{*}$ such that $\operatorname{dim}\left(\mathfrak{g}^{x}\right)^{\bar{a}}=\mathrm{rk} \mathfrak{g}$ and $(\mathbb{k} a+\mathbb{k} x) \cap \mathfrak{g}_{\text {sing }}^{*} \subset \mathbb{k} x$ is a highly non-trivial task.

2.2. The rôle of nilpotent orbits. Let $\mathcal{N}$ denote the set of nilpotent elements of $\mathfrak{g} \simeq \mathfrak{g}^{*}$. Any $G$-orbit in $\mathcal{N}$ is said to be nilpotent. As is well known, $\mathcal{N} / G$ is finite and any $G$-orbit in $\mathfrak{g}$ can be contracted to a nilpotent one, see a construction below. This turns out to be extremely helpful in the theory of complete algebras. 
Proposition 2.5. Let $\mathcal{A} \subset \mathcal{S}(\mathfrak{g})$ be a homogeneous subalgebra. If $\operatorname{tr} \cdot \operatorname{deg}\left(\left.\mathcal{A}\right|_{G e}\right)=\frac{1}{2} \operatorname{dim}(G e)$ for each nilpotent element $e \in \mathfrak{g}^{*}$, then $\operatorname{tr} \cdot \operatorname{deg}\left(\left.\mathcal{A}\right|_{G x}\right)=\frac{1}{2} \operatorname{dim}(G x)$ for each $x \in \mathfrak{g}^{*}$.

Proof. The statement is vacuous for nilpotent orbits. Assume therefore that $x \notin \mathcal{N}$. Set $Y=\overline{\mathbb{k}^{\times}(G x)}$. This is a conical subvariety of $\mathfrak{g}^{*}$ and $\operatorname{dim} Y=\operatorname{dim} G x+1$. By the method of associated cones introduced and developed in [BK79, §3], there is an orbit $G e \subset Y \cap \mathcal{N}$ such that $\operatorname{dim}(G e)=\operatorname{dim}(G x)$. Observe that $d_{x} \mathcal{A}=d_{t x} \mathcal{A}$ for each non-zero $t \in \mathbb{k}$, because $\mathcal{A}$ is homogeneous. Therefore

$$
\max _{y \in Y} \operatorname{dim} d_{y} \mathcal{A}=\max _{x^{\prime} \in G x} \operatorname{dim} d_{x^{\prime}} \mathcal{A}
$$

and in particular

$$
\max _{x^{\prime} \in G x} \operatorname{dim} d_{x^{\prime}} \mathcal{A} \geqslant \max _{e^{\prime} \in G e} \operatorname{dim} d_{e^{\prime}} \mathcal{A} .
$$

A possible way to conclude the proof would be to calculate $\operatorname{dim}\left(d_{x} \mathcal{A} \cap \mathfrak{g}^{x}\right)$ and $\operatorname{dim}\left(d_{e} \mathcal{A} \cap\right.$ $\left.\mathfrak{g}^{e}\right)$. For instance, if $\mathcal{A}=\mathcal{F}_{a}$ is an MF-subalgebra with $a \in \mathfrak{g}_{\text {reg }}^{*}$, then $\operatorname{dim}\left(d_{y} \mathcal{A} \cap \mathfrak{g}^{y}\right)=l$ for any $y \in \mathfrak{g}^{*}$ by Lemma 2.1 and there is nothing else to show. But in case of a general $\mathcal{A}$, our approach is different.

Since $x$ is not nilpotent, there is a homogeneous non-constant polynomial $H \in \mathcal{S}(\mathfrak{g})^{\mathfrak{g}}$ such that $c=\operatorname{deg} H>0$ and $H(x) \neq 0$. Assume that homogeneous elements $\tilde{a}_{1}, \ldots, \tilde{a}_{m} \in$ $\mathcal{A}$ are algebraically independent on $G e$, but dependent on $G x$. Without violating these assumptions, replace each $\tilde{a}_{i}$ with $a_{i}=\tilde{a}_{i}^{c}$. Set $c_{i}=\operatorname{deg} \tilde{a}_{i}$. Let $\mathbf{Q}$ be a non-trivial relation among $\left.a_{i}\right|_{G x}$. Then $\mathrm{Q}\left(\frac{a_{1}}{H^{c_{1}}}, \ldots, \frac{a_{m}}{H^{c_{m}}}\right)=0$ on $\mathbb{k}^{\times}(G x)$. Multiplying this equality by a suitable power of $H$ and restricting to $G e$, where $H$ vanishes, we obtain a non-trivial relation among $\left.a_{1}\right|_{G e}, \ldots,\left.a_{m}\right|_{G e}$. A contradiction! Thus,

$$
\operatorname{tr} \cdot \operatorname{deg}\left(\left.\mathcal{A}\right|_{G e}\right) \leqslant \operatorname{tr} \cdot \operatorname{deg}\left(\left.\mathcal{A}\right|_{G x}\right)
$$

and the result follows.

The sheets of $\mathfrak{g}$ are the irreducible components of the locally closed subsets $X^{(d)}=\{\xi \in$ $\mathfrak{g} \mid \operatorname{dim}(G \xi)=d\}$ for all $d$. Let $G e$ be a nilpotent orbit in $\overline{\mathbb{K}^{\times}(G x)}$ with $\operatorname{dim}(G e)=\operatorname{dim}(G x)$. Then $G e$ is a nilpotent orbit in each sheet $S$ containing $G x$. By a fundamental result of Borho and Kraft, each sheet contains a unique nilpotent orbit [BK79, Sect. 5.8. Kor.(a)]. Therefore the associated cone of $G x$, i.e., the variety $\overline{\mathbb{k}^{\times}(G x)} \backslash \mathbb{k}^{\times}(\overline{G x})$, is irreducible and the above-mentioned orbit $G e$ is unique. Equation (2.5) leads to the following statement.

Corollary 2.6. Suppose that a homogeneous Poisson-commutative subalgebra $\mathcal{A} \subset \mathcal{S}(\mathfrak{g})$ is complete on a nilpotent orbit $G e$. Then $\mathcal{A}$ is complete on any orbit $G x$ such that $G x$ and $G e$ lie in one and the same sheet. 
Remark 2.7. Proposition 2.5 has a rather amusing application. For, our considerations with nilpotent orbits easily recover the main result of a recent preprint [CRR], which asserts that the MF-subalgebra $\mathcal{F}_{a}$ with $a \in \mathfrak{g}_{\text {reg }}^{*}$ is complete on each $G x \subset \mathfrak{g}_{\text {reg }}^{*}$. Note that a more general result is already contained in Theorem 2.4, but the argument for the regular elements $x$ only can be made astonishingly simple and short. It uses neither Slodowy slices nor the Kostant section. Namely, let $\{e, h, f\}$ be a principal $\mathfrak{s l}_{2}$-triple in $\mathfrak{g}$. Assume that $\mathcal{F}_{a}$ is not complete on $G x$. Then $\mathcal{F}_{a}$ is not complete on $G e$, see Proposition 2.5. Then $\mathcal{F}_{e}$ is not complete on $G a$ by Theorem 2.3. Then $\mathcal{F}_{e}$ is not complete on $G e$ again by Proposition 2.5. However, this is absurd, since $\langle e, f\rangle_{\mathrm{k}} \subset \mathfrak{g}_{\mathrm{reg}}^{*} \cup\{0\}$ and $\operatorname{dim} d_{f} \mathcal{F}_{e}=\boldsymbol{b}(\mathfrak{g})$, cf. Corollary 1.5.

In what follows, $e$ stands for an arbitrary nilpotent element of $\mathfrak{g}$.

Proposition 2.8. There is a non-empty open subset $U \subset \mathfrak{g}_{\mathrm{reg}}^{*}$ such that for any $a \in U$, the MF-subalgebra $\mathcal{F}_{a}$ is complete on every adjoint orbit.

Proof. Recall that $\mathcal{N} / G$ is finite. For each $G e \subset \mathcal{N}$, the subset

$$
U(e)=\left\{a \in \mathfrak{g}_{\mathrm{reg}}^{*} \mid \mathcal{F}_{a} \text { is complete on } G e\right\}
$$

is non-empty and open in $\mathfrak{g}^{*}$ [B91, Thm 3.2]. Let $U$ be the intersection of $U(e)$ taken over all nilpotent orbits. Then $U \neq \varnothing$ is open in $\mathfrak{g}^{*}$. For any $a \in U$, the MF-subalgebra $\mathcal{F}_{a}$ is complete on every nilpotent and hence on every adjoint orbit, see Proposition 2.5.

Proposition 2.8 opens ample possibilities for further generalisations. It would be nice to prove that, for each $a \in \mathfrak{g}_{\text {reg }}^{*}, \mathcal{F}_{a}$ is complete on any adjoint orbit.

2.3. Complete families. For $a \in \mathfrak{g}_{\text {reg, }}^{*}$ Theorem 2.4 implies that $\operatorname{tr} . \operatorname{deg} \mathcal{F}_{a}=\boldsymbol{b}(\mathfrak{g})$ and thereby the generators $\partial_{a}^{k} H_{i} \in \mathcal{F}_{a}$ with $1 \leqslant i \leqslant l$ and $0 \leqslant k<\operatorname{deg} H_{i}$ are algebraically independent. Suppose that $\operatorname{dim} d_{x}\left(\mathcal{F}_{a}\right)=\mathrm{rk} \mathfrak{g}+\frac{1}{2} \operatorname{dim}(G x)$ for some $x \in \mathfrak{g}^{*}$. If $x$ is regular as well, one restricts the polynomials $\partial_{a}^{k} H_{i}$ with $1 \leqslant i \leqslant l$ and $0<k<\operatorname{deg} H_{i}$ to $G x$ in order to obtain a complete family in involution. Suppose now that $\operatorname{dim}(G x)<\operatorname{dim} \mathfrak{g}-\mathrm{rk} \mathfrak{g}$. Then some other generators of $\mathcal{F}_{a}$ become redundant on $G x$. A natural question is, which ones? There is a simple answer in types A and C.

Suppose that $\mathfrak{g}$ is either $\mathfrak{g l}_{l}, \mathfrak{s l}_{l+1}$, or $\mathfrak{s p}_{2 l}$. As generating symmetric invariants $H_{1}, \ldots, H_{l}$ we take coefficients of the characteristic polynomial. Assume that $\operatorname{deg} H_{i}>\operatorname{deg} H_{j}$ whenever $i>j$. Set $d_{i}=\operatorname{deg} H_{i}$. According to [MY17, Sect. 2], $\mathcal{F}_{x}$ is a free algebra with a set $\left\{\partial_{x}^{k} H_{i} \mid 1 \leqslant i \leqslant i, 0 \leqslant k \leqslant s(i)\right\}$ of algebraically independent generators. Moreover, the numbers $s(i)$ depend only on the partition of $e$, where $G e$ is the dense orbit in the associated cone of Gx. The dependence is very explicit, see [MY17, Sect. 4]. We note also that $\partial_{e}^{s(i)} H_{i} \in \mathcal{S}\left(\mathfrak{g}^{e}\right)$ and that $\partial_{e}^{k} H_{i}=0$ if $k>s(i)$. 
Proposition 2.9. Suppose that $\mathfrak{g}$ is of type $\mathrm{A}$ or $\mathrm{C}$. Assume that $\mathcal{F}_{a}$ with $a \in \mathfrak{g}_{\text {reg }}^{*}$ is complete on $G e$. Then the restrictions of $\partial_{a}^{k} H_{i}$ with $d_{i}>k>d_{i}-s(i)$ to $G x$ is a complete family in involution.

Proof. By virtue of Proposition 2.5, it suffices to prove the assertion for Ge. According to (2.2), the differential $d_{e}\left(\partial_{a}^{k} H_{i}\right)$ is equal to $d_{a}\left(\partial_{e}^{d_{i}-k-1} H_{i}\right)$ up to a non-zero rational scalar. If $d_{i}-k-1>s(i)$, then $\partial_{e}^{d_{i}-k-1} H_{i}=0$ and hence also $d_{e}\left(\partial_{a}^{k} H_{i}\right)=0$; if $d_{i}-k-1=s(i)$, then $d_{e}\left(\partial_{a}^{k} H_{i}\right) \in \mathfrak{g}^{e}$. The same statements for the differentials hold at each point $e^{\prime} \in G e$. If $F \in \mathcal{S}(\mathfrak{g})$ and $d_{e^{\prime}} F \in \mathfrak{g}^{e^{\prime}}$ for each $e^{\prime} \in G e$, then $\left.F\right|_{G e}$ is a constant, if in addition $F$ is homogeneous, then $\left.F\right|_{G e}=0$. Thus, the polynomials $\partial_{a}^{k} H_{i}$ with $k \leqslant d_{i}-s(i)-1$ restrict to zero on $G e$. The number of the remaining elements, $\partial_{a}^{k} H_{i}$ with $d_{i}>k>d_{i}-s(i)$, is equal to tr.deg $\mathcal{F}_{e}-l=\frac{1}{2} \operatorname{dim}(G e)$, see [MY17, Sect. 2].

Example 2.10. Take $\mathfrak{g}=\mathfrak{g l}_{n}$. A nilpotent orbit $G e \subset \mathfrak{g}$ is determined by a partition $\boldsymbol{r}=$ $\left(r_{1}, \ldots, r_{t}\right)$ of $n$, where $r_{1} \geqslant r_{2} \geqslant \ldots \geqslant r_{t}>0$ are the sizes of Jordan blocks of $e$. We then set $\mathcal{O}(\boldsymbol{r}):=G e$. The numbers $s(i)$ appeared in [PPY, Thm 4.2] as the degrees of certain generators of $\mathcal{S}\left(\mathfrak{g}^{e}\right)^{\mathfrak{g}^{e}}$, cf. [MY17, Lemma 1.5]. They are uniquely defined by the conditions

$$
\sum_{j=1}^{s(i)-1} r_{j}<i \leqslant \sum_{j=1}^{s(i)} r_{j} .
$$

To give a graphic presentation of the complete family of Proposition 2.9, we first arrange the polynomials $\partial_{a}^{k} H_{i}$ into the left justified Young tableau, where $H_{n}, \ldots, H_{1}$ form the first (top) row, $\partial_{a} H_{n}, \ldots, \partial_{a} H_{2}$ - the second row, and so on until the last (bottom) row, where just $\partial_{a}^{n-1} H_{n}$ stands in the left column. The resulting diagram has consecutive rows of size $(n, n-1, \ldots, 1)$, hence it has $n(n+1) / 2=\boldsymbol{b}(\mathfrak{g})$ boxes.

Next, we define a certain colour pattern corresponding to $\mathcal{O}(\boldsymbol{r})$. This pattern is going to be used in Section 4. The recipe is the following:

$\diamond$ in the top row paint the last (looking from the left) $r_{1}$ boxes in red and all boxes below them in green;

$\diamond$ in the second row find the rightmost box that is not green, starting from it make a stripe of red boxes of length $r_{2}$, paint all the boxes below the stripe in green;

$\diamond$ if the first $m-1$ rows are painted and $r_{m}>0$, then find the rightmost box in the $m$-th row that is not green; starting from it make a stripe of red boxes of length $r_{m}$, and paint all the boxes below the stripe in green.

The green boxes depict the complete family of Proposition 2.9 and therefore there are $\frac{1}{2} \operatorname{dim}(G e)$ of them. It is easily seen that we have $n$ red boxes. These boxes are going to be used in Section 4.

The colour patterns corresponding to the partitions $(3,2,1),(4,1)$, and $(2,2,2,1)$ are presented below. 

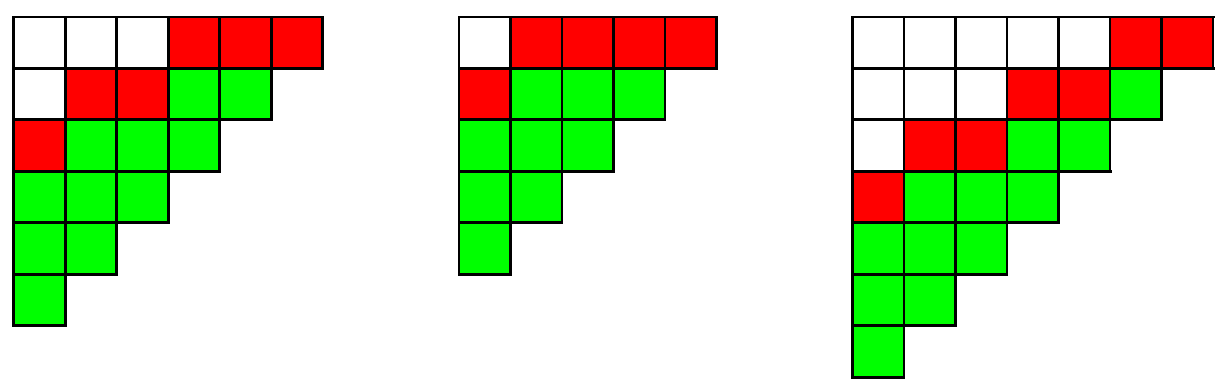

\section{FLAG VARIETIES AND COISOTROPIC ACTIONS}

Suppose for a while that $G$ is a complex reductive group. Let $B \subset G$ be a Borel subgroup, $T(\mathbb{C}) \subset B$ a maximal torus in $G, P \subset G$ a parabolic containing $B$. Fix also a maximal compact subgroup $K \subset G$ such that $T=K \cap T(\mathbb{C})$ is a maximal torus in $K$. Set $\mathfrak{k}=$ Lie $K$, $\mathfrak{t}=$ Lie $T$. Let further $V_{\lambda}$ be a finite-dimensional simple $G$-module with a highest weight vector $v_{\lambda}$. Standard facts are that $G / B \simeq K / T$ and $G / P \simeq K / L$, where $L=P \cap K$, and the (real) symplectic structure on $G / P=G\left\langle v_{\lambda}\right\rangle \subset \mathbb{P} V_{\lambda}$ is the same as on the (co)adjoint orbit $K \lambda \subset \mathfrak{k}^{*}$. This is one of the reasons, why integrable systems ( $\sim$ complete families in involution) on adjoint orbits of compact groups are of particular interest.

Definition 1 can be reformulated for any symplectic manifold or variety $M$. If $M$ is not algebraic, then one has to consider smooth (or differentiable) functions and replace "algebraically independent" with "functionally independent". In what follows, we write simply "a complete family" instead of "a complete family in involution". Strictly speaking, an integrable system includes also a choice of a Hamiltonian, a function $\boldsymbol{H}$ on $M$ that Poisson-commutes with a complete family. Fortunately, an arbitrary element of a complete family can be chosen as $\boldsymbol{H}$.

The most famous example of a complete family on a flag variety is the Gelfand-Tsetlin system of Guillemin-Sternberg in the $\mathrm{U}_{n}$-case [GS83], the $\boldsymbol{\lambda}$-system in our terminology, see the Introduction and Section 4 for its description. There is also a direct analogue in the orthogonal case [GS83'] and a symplectic variation due to Harada [H06]. We demonstrate below that MF-subalgebras lead to integrable systems on flag varieties. Our construction is independent of the type of $G$.

Although we have assumed so far that $\overline{\mathbb{k}}=\mathbb{k}$, MF-subalgebras can be defined in the same way over $\mathbb{Q}$ for the rational forms of $\mathfrak{g}$, as well as for the real forms. In particular, the method works for $\mathfrak{k}$. This was already clear to Mishchenko and Fomenko [MF78]. Observe that $\mathcal{S}(\mathfrak{k})^{\mathfrak{k}} \otimes_{\mathbb{R}} \mathbb{C}=\mathcal{S}(\mathfrak{g})^{\mathfrak{g}}$. Choose a parameter $a \in \mathfrak{k}^{*}$ and let $\mathcal{F}_{a} \subset \mathcal{S}(\mathfrak{k})$ be the MF-subalgebra associated with $a$. Then $\mathcal{F}_{a}(\mathbb{C})=\mathcal{F}_{a} \otimes_{\mathbb{R}} \mathbb{C}$ is the complex MF-subalgebra of $\mathcal{S}(\mathfrak{g})$ associated with $a$, where $a$ is regarded as a complex valued linear function on $\mathfrak{g}$.

Let $\{e, h, f\} \subset \mathfrak{g}$ be a principal $\mathfrak{s l}_{2}$-triple such that

$$
\langle e, h, f\rangle_{\mathbb{C}} \cap \mathfrak{k}=\langle i h, f-e, i f+i e\rangle_{\mathbb{R}} \text { and } i h \in \mathfrak{t} .
$$


Note that $\mathfrak{g}^{x}=\mathfrak{k}^{x} \otimes_{\mathbb{R}} \mathbb{C}$ for any $x \in \mathfrak{k}^{*}$. Hence $\mathfrak{k}_{\text {reg }}^{*} \subset \mathfrak{g}_{\text {reg }}^{*}$.

Proposition 3.1. Take $a \in \mathfrak{k}_{\text {reg }}^{*}$. Then the real MF-subalgebra $\mathcal{F}_{a}$ is complete on any orbit $K x \in \mathfrak{k}^{*}$ and therefore on any flag variety $G / P=G\left\langle v_{\lambda}\right\rangle$. If we choose $a=f-e \in \mathfrak{k} \simeq \mathfrak{k}^{*}$, then $\operatorname{dim} d_{x}\left(\left.\mathcal{F}_{a}\right|_{K x}\right)=\frac{1}{2} \operatorname{dim}(K x)$ for every $x \in \mathfrak{t}^{*}$.

Proof. All elements of $\mathfrak{k}$ are semisimple. By Theorem 2.4, $\mathcal{F}_{a}(\mathbb{C})$ is complete on $G x \subset \mathfrak{g}^{*}$ if $a \in \mathfrak{k}_{\text {reg }}^{*}$ and $x \in \mathfrak{k}^{*}$. The equality $\operatorname{dim} d_{y}\left(\left.\mathcal{F}_{a}(\mathbb{C})\right|_{G x}\right)=\frac{1}{2} \operatorname{dim}(G x)$ holds for each $y \in U$, where $U \subset G x$ is a non-empty Zariski open subset. In the complex Zariski topology, $K x$ is dense in $G x$. Hence $U \cap K x \neq \varnothing$. By a standard linear algebra argument, for any $x \in \mathfrak{k}^{*}$, we have $d_{x} \mathcal{F}_{a} \otimes_{\mathbb{R}} \mathbb{C}=d_{x} \mathcal{F}_{a}(\mathbb{C})$. Thus, $\mathcal{F}_{a}$ is complete on $K x$.

If $a=f-e$ and $x \in \mathfrak{t}^{*}$, then $\operatorname{dim} d_{x}\left(\left.\mathcal{F}_{a}(\mathbb{C})\right|_{G x}\right)=\frac{1}{2} \operatorname{dim}(G x)$ according to the proof of Theorem 2.4. Hence here $\operatorname{dim}_{\mathbb{R}} d_{x}\left(\left.\mathcal{F}_{a}\right|_{K x}\right)=\frac{1}{2} \operatorname{dim}_{\mathbb{R}}(K x)$.

The Gelfand-Tsetlin system of Guillemin-Sternberg is complete on each adjoint orbit of $\mathrm{U}_{n}$. The key point here is that the action of $\mathrm{U}_{n-1}$ on a (co)adjoint orbit of $\mathrm{U}_{n}$ is coisotropic, which is formulated in [GS83']. Guillemin and Sternberg prove this assertion if the orbit in question is regular, the non-regular case being illustrated through examples. The statement, for both $\mathrm{U}_{n}$ and $\mathrm{SO}_{n}(\mathbb{R})$, is attributed to Heckman [H82], see e.g. [GS83', p. 225]. Below, we give a modern perspective on the matter and show that the non-regular case follows easily from the regular one.

3.1. Coisotropic actions. The symplectic manifolds (or varieties) $(M, \omega)$ endowed with a coisotropic action of a group are also known as the "multiplicity-free spaces" [GS84, HW90]. The starting point is a Hamiltonian action of a group $Q$ on $M$, see e.g. [GS80, Sect. 2] for the definition. In this section, we assume that either $M$ is a smooth variety over $\mathbb{k}$ and $Q$ is an affine algebraic group defined over $\mathbb{k}$ or $M$ is a homogeneous space of a compact real group $K$ and $Q$ is a compact real group. In both cases, $M$ is assumed to be irreducible.

Associated with the Hamiltonian action of $Q$, there is a moment map $\mu=\mu_{Q}: M \rightarrow \mathfrak{q}^{*}$, see [GS80, Sect. 3]. In this paper, we are interested only in cases, where the moment map is defined globally. The elements of $\mu^{*}(\mathcal{S}(\mathfrak{q}))$ are functions on $M$ and they are called either Noether integrals or collective functions. We have either $\mu^{*}(\mathcal{S}(\mathfrak{q})) \subset \mathbb{k}[M]$ or $\mu^{*}(\mathcal{S}(\mathfrak{q})) \subset \mathbb{R}[M]$, depending on the context. The name "Noether integrals" is justified by the following theorem of Emmy Noether: $\left\{F, \mu^{*}(\mathcal{S}(\mathfrak{q}))\right\}=0$ for each $Q$-invariant function $F$ on $M$. The term "collective functions" is introduced in [GS83'].

Let $\mathbb{L}$ denote either $\mathbb{k}$ or $\mathbb{R}$. Write $\mathbb{L}(M)^{Q}$ for the field of $Q$-invariant rational functions on $M$. For $x \in M$, set $\mathfrak{q} x=T_{x}(Q x)$. 
Definition 2. A Hamiltonian action of $Q$ on $M$ is coisotropic if $(\mathfrak{q} x)^{\perp} \subset(\mathfrak{q} x)$ for generic $x \in M$, where the orthogonal complement is taken w.r.t. the symplectic form $\omega_{x}$.

Since $\omega_{x}$ is non-degenerate, the condition $(\mathfrak{q} x)^{\perp} \subset(\mathfrak{q} x)$ is equivalent to that

$$
\omega_{x} \text { vanishes on }(\mathfrak{q} x)^{\perp} \text {. }
$$

There are many equivalent conditions that define coisotropic actions, see e.g. [GS83', Sect. 2]. Some of them are presented below.

The Poisson structure $\pi$ on $M$ is given by $\pi(x)=\left(\omega_{x}^{-1}\right)^{t}$ at $x \in M$. Here $\omega_{x}$ is a skewsymmetric form on $T_{x} M$ and $\pi(x)$ is a skew-symmetric form on $T_{x}^{*} M$. By duality between $\omega$ and $\pi$, we have

$$
\left.\omega_{x}\right|_{(\mathfrak{q} x)^{\perp}}=\left.0 \Longleftrightarrow \pi(x)\right|_{\operatorname{Ann}(\mathfrak{q} x)}=0 .
$$

Let $F$ be a $Q$-invariant rational function on $M$ such that $d_{x} F$ is defined. Then $d_{x} F$ vanishes on $\mathfrak{q} x$, i.e., $d_{x} F \in \operatorname{Ann}(\mathfrak{q} x)$. By the Rosenlicht theorem, see e.g. [VP89, Thm 2.3], the rational $Q$-invariants on $M$ separate generic $Q$-orbits. Hence there is a non-empty subset $U \subset M$ such that for each $y \in U$ there are rational functions $F_{1}, \ldots, F_{m} \in \mathbb{L}(M)^{Q}$ satisfying $\left\langle F_{i} \mid 1 \leqslant i \leqslant m\right\rangle_{\mathbb{L}}=\operatorname{Ann}(\mathfrak{q} y)$. Therefore (3.1) holds generically if and only if

$$
\mathbb{L}(M)^{Q} \text { is Poisson commutative. }
$$

If $\operatorname{tr} \cdot \operatorname{deg} \mathbb{L}[M]^{Q}=\operatorname{tr} \cdot \operatorname{deg} \mathbb{L}(M)^{Q}$, then (3.3) is equivalent to

$$
\mathbb{L}[M]^{Q} \text { is Poisson commutative. }
$$

Note that in the compact setting, the regular invariants $\mathbb{R}[M]^{Q}$ separate all $Q$-orbits. Further conditions involve $\mu$.

Observe that $\operatorname{ker}\left(d_{x} \mu\right)=(\mathfrak{q} x)^{\perp}$, see e.g. [GS83', Eq. (1.6)] or [Vi01, Eq. (56)]. Thus,

$$
(\mathfrak{q} x)^{\perp} \subset \mathfrak{q} x \Longleftrightarrow\left(d_{x} \mu\right)^{-1}(\mathfrak{q} \mu(x))=\mathfrak{q} x .
$$

Suppose that $\operatorname{dim} M=2 n$ and $F_{1}, \ldots, F_{n}$ is a complete family on $M$ consisting of Noether integrals, i.e., $F_{i} \in \operatorname{Im} \mu^{*}$ for each $i$. For $x \in M$, set $L(x)=\left\langle d_{x} F_{i} \mid 1 \leqslant i \leqslant n\right\rangle_{\mathbb{L}}$. Then $\left.\pi(x)\right|_{L(x)}=0$ and $L(x)$ is ortogonal to $d_{x}\left(\mathbb{L}(M)^{Q}\right)$ w.r.t. $\pi(x)$. If $x$ is generic, then $L(x)$ is a Lagrangian subspace of $T_{x}^{*} M$ w.r.t. $\pi(x)$ and $d_{x}\left(\mathbb{L}(M)^{Q}\right)=\operatorname{Ann}(\mathfrak{q} x)$. For such an $x$, we have $\operatorname{Ann}(\mathfrak{q} x) \subset L(x)$ and hence $\pi(x)$ vanishes on $\operatorname{Ann}(\mathfrak{q} x)$. Therefore, it follows from (3.2), see also the theorem in [GS83', Sect. 2], that the following assertion is true:

(NF) there is a complete family on $M$ consisting of Noether integrals only if the action of $Q$ on $M$ is coisotropic.

Theorem 3.2 ([GS83']). The action of $\mathrm{U}_{n-1}$ on any adjoint orbit of $\mathrm{U}_{n}$ is coisotropic. 
Proof. Set $Q=\mathrm{U}_{n-1}, M=\mathrm{U}_{n} x \subset \mathfrak{u}_{n}$. Suppose first that $M$ is a regular $\mathrm{U}_{n}$-orbit. Take $y \in \mu(M)$. Then $y$ is a regular point of $\mathfrak{q}\left[\mathrm{GS83}\right.$ ', Sect. 4] and $Q_{y}$ acts on $\mu^{-1}(y)$ transitively if $y \in \mu(M)$ is generic [GS83', Eq. (2.5)]. Combining these facts with (3.5), we obtain that (3.1) holds at each point $x \in \mu^{-1}(y)$. It is also true that $\left\{\mathcal{S}\left(\mathfrak{u}_{n}\right)^{Q}, \mathcal{S}\left(\mathfrak{u}_{n}\right)^{Q}\right\}$ vanishes on $M$, cf. (3.4). Since this holds for any regular orbit, $\mathcal{S}\left(\mathfrak{u}_{n}\right)^{Q}$ is Poisson-commutative.

Next, let $M \subset \mathfrak{u}_{n}$ be an arbitrary adjoint orbit. Since $Q$ is compact,

$$
\mathbb{R}(M)^{Q}=\operatorname{Quot}\left(\mathbb{R}[M]^{Q}\right)
$$

and $\mathbb{R}[M]^{Q}$ is the restriction of $\mathcal{S}\left(\mathfrak{u}_{n}\right)^{Q}$ to $M$. In particular, $\mathbb{R}[M]^{Q}$ is Poisson-commutative and this implies that (3.1) holds for genetic $x \in M$.

Theorem 3.2 combined with an inductive argument of [GS83', (2.9)], yields the following assertion.

Corollary 3.3 ([GS83']). The integrable system of [GS83], the type A $\boldsymbol{\lambda}$-system in our terminology, is complete on any adjoint orbit of $\mathrm{U}_{n}$.

A similar inductive argument applies in the orthogonal case, too. Actually, Section 3.2 contains a thorough discussion of the fact that the action of $\mathrm{SO}_{n-1}(\mathbb{R})$ on every adjoint orbit of $\mathrm{SO}_{n}(\mathbb{R})$ is coisotropic.

The "multiplicity-free spaces" of [GS84, HW90] are related to multiplicity-free decompositions and spherical varieties. An algebraic $\mathbb{k}$-variety $X$ acted upon by a reductive group $G$ is said to be spherical, if a Borel subgroup $B \subset G$ acts on $X$ with an open orbit.

Suppose that $M$ is Kähler and $Q$ is a compact real group. Then the action of $Q$ on $M$ is coisotropic if and only if

(SC) $M$ is a spherical $Q(\mathbb{C})$-variety [HW90, Sect. 6].

A complex flag variety $G / P$ is definitely Kähler. Take $Q \subset K \subset G$. If $G / B$ is spherical w.r.t. $Q(\mathbb{C})$, then $G / P$ is also a spherical $Q(\mathbb{C})$-variety for each parabolic $P$. This is another way to see that if a generic adjoint orbit of $K$ is coisotropic w.r.t. $Q$, then each adjoint orbit of $K$ is also $Q$-coisotropic.

3.2. Strong Gelfand pairs. Among pairs of reductive groups $H \subsetneq G$, two occupy the most prominent position. These are the strong Gelfand pairs $\left(\mathrm{GL}_{n}(\mathbb{k}), \mathrm{GL}_{n-1}(\mathbb{k})\right)$ and $\left(\mathrm{SO}_{n}(\mathbb{k}), \mathrm{SO}_{n-1}(\mathbb{k})\right)$. Up to local isomorphisms, products, products with $(H, H)$, and pairs $\left(\mathbb{k}^{\times},\{e\}\right)$, these are the only strong Gelfand pairs, see [Kr76] and [H82, Sect. 4].

Strong Gelfand pairs can be characterised by a host of equivalent conditions. Below we present a selection of these conditions:

(Sph1) the homogeneous space $(G \times H) / H$ is a spherical $(G \times H)$-variety;

(Sph2) $G / B$ is a spherical $H$-variety; 
(Br) each irreducible finite-dimensional representation $V_{\lambda}$ of $G$ decomposes without multiplicities under the action of $H$;

(Com) the algebra $\mathcal{U}(\mathfrak{g})^{\mathfrak{h}}$ is commutative;

$(\mathrm{PCm})$ the algebra $\mathcal{S}(\mathfrak{g})^{\mathfrak{l}}$ is Poisson-commutative;

(Cois) the action of $H$ on each closed orbit $G x \subset \mathfrak{g}^{*}$ is coisotropic;

$($ DCn $) \mathcal{S}(\mathfrak{g})^{\mathfrak{h}}=\operatorname{alg}\left\langle\mathcal{S}(\mathfrak{g})^{\mathfrak{g}}, \mathcal{S}(\mathfrak{h})^{\mathfrak{h}}\right\rangle$;

(CtB) the action of $H$ on $T^{*}(G / P)$ is coisotropic for each parabolic $P \subset G$.

It is a classical fact that the pairs $\left(\mathrm{GL}_{n}(\mathbb{k}), \mathrm{GL}_{n-1}(\mathbb{k})\right)$ and $\left(\mathrm{SO}_{n}(\mathbb{k}), \mathrm{SO}_{n-1}(\mathbb{k})\right)$ satisfy $(\mathrm{Br})$. It took a long time and many papers to prove the equivalences of the above conditions. Below is a brief outline.

Remark 3.4. [Arguments for the equivalences.] The fact that (Sph1) $\Leftrightarrow(\mathrm{Sph} 2)$ is observed in [AP02], see Eq. (5) on page 26 therein.

Both equivalences $(\mathrm{Sph} 1) \Leftrightarrow(\mathrm{Br})$ and $(\mathrm{Sph} 2) \Leftrightarrow(\mathrm{Br})$ are results of [VK78]. The action of $H$ on the flag variety $G\left\langle v_{\lambda}\right\rangle \subset \mathbb{P} V_{\lambda}$ is spherical if and only if each $V_{n \lambda}$ with $n \in \mathbb{N}$ decomposes without multiplicities under the action of $H$. In the affine case, $(G \times H) / H$ is a spherical $(G \times H)$-variety if and only if $\operatorname{dim}\left(V_{\lambda} \otimes V_{\mu}\right)^{H} \leqslant 1$ for all irreducible finite dimensional $G$-modules $V_{\lambda}$ and $H$-modules $V_{\mu}$.

A simple proof for the equivalence $(\mathrm{Br}) \Leftrightarrow(\mathrm{Com})$ is given in [J01].

Since $\mathcal{S}(\mathfrak{g})^{\mathfrak{h}}=\operatorname{gr}\left(\mathcal{U}(\mathfrak{g})^{\mathfrak{h}}\right)$, we have $($ Com $) \Rightarrow(P C m)$.

The implication $(\mathrm{PCm}) \Rightarrow(\mathrm{Sph} 1)$ can be extracted from the proof of [Kn90, Satz 2.3], see the implication $\left(2^{\prime} \Rightarrow 3\right)$ therein. In [Kn90, Satz 2.3], it is shown that $(\mathrm{PCm}) \Leftrightarrow($ Cois $) \Leftrightarrow$ $(\mathrm{DCn})$. That proof exploits the classification of strong Gelfand pairs. Below we give an alternative, classification-free argument, see Theorem 3.6.

Observe that the implication (DCn) $\Rightarrow($ Com $)$ is almost trivial. Let $\varpi: \mathcal{S}(\mathfrak{g}) \rightarrow \mathcal{U}(\mathfrak{g})$ be the symmetrisation map. It is a homomorphism of $G$-modules. Thereby $\mathcal{U}(\mathfrak{g})^{\mathfrak{h}}=\varpi\left(\mathcal{S}(\mathfrak{g})^{\mathfrak{h}}\right)$. Suppose that $(D C n)$ holds. Then $\mathcal{S}(\mathfrak{g})^{\mathfrak{h}}$ is generated by $\mathcal{S}(\mathfrak{h})^{\mathfrak{h}}$ as an $\mathcal{S}(\mathfrak{g})^{\mathfrak{g}}$-module. Therefore $\mathcal{U}(\mathfrak{g})^{\mathfrak{h}}$ is generated by $\mathcal{U}(\mathfrak{h})^{\mathfrak{h}}$ as a $\mathcal{U}(\mathfrak{g})^{\mathfrak{g}}$-module. Since $\left[\mathfrak{U}(\mathfrak{h})^{\mathfrak{h}}, \mathcal{U}(\mathfrak{h})^{\mathfrak{h}}\right]=0$, the condition (Com) holds.

Finally, the equivalence $(\mathrm{CtB}) \Leftrightarrow(\mathrm{Sph} 2)$ follows from [Kn90', Satz 7.1], see also [Vi01, Chapter 2, §3] and in particular Theorem 2 therein.

An open subset $U$ of an irreducible algebraic variety $X$ is said to be big if $\operatorname{dim} X \backslash U \leqslant$ $\operatorname{dim} X-2$.

Lemma 3.5. Let $H \subset G$ be a reductive subgroup. Set $\mathcal{C}_{1}=\operatorname{alg}\left\langle\mathcal{S}(\mathfrak{g})^{\mathfrak{g}}, \mathcal{S}(\mathfrak{h})^{\mathfrak{h}}\right\rangle$. Then $\mathcal{C}_{1}$ is an algebraically closed subalgebra of $\mathcal{S}(\mathfrak{g})$. 
Proof. If $\mathfrak{h}$ contains a non-trivial ideal of $\mathfrak{g}$, we can replace $H$ by a smaller subgroup without altering $\mathfrak{C}_{1}$. Therefore assume that $\mathfrak{h}$ contains no non-trivial ideals of $\mathfrak{g}$. Then $\mathfrak{C}_{1}$ is generated by homogenous algebraically independent elements $\left\{\boldsymbol{c}_{1}, \ldots, \boldsymbol{c}_{r}\right\}$ such that $\mathcal{S}(\mathfrak{g})^{\mathfrak{g}}=\mathbb{k}\left[\boldsymbol{c}_{1}, \ldots, \boldsymbol{c}_{l}\right]$ and $\mathcal{S}(\mathfrak{h})^{\mathfrak{h}}=\mathbb{k}\left[\boldsymbol{c}_{l+1}, \ldots, \boldsymbol{c}_{r}\right]$, see $\left[K n 90\right.$, Satz 2.1]. For $x \in \mathfrak{g}^{*}$, set $\bar{x}=\left.x\right|_{\mathfrak{h}}$. In view of the Kostant regularity criterion (2.1), we have $\operatorname{dim} d_{x} \mathcal{C}_{1}=r$ if and only if

$$
x \in \mathfrak{g}_{\text {reg }}^{*}, \bar{x} \in \mathfrak{h}_{\text {reg }}^{*} \text {, and } \mathfrak{g}^{x} \cap \mathfrak{h}^{\bar{x}}=0 .
$$

The first two conditions hold on big open subsets. The third one holds if and only if $\mathfrak{h}^{x}=0$. Write $x=\bar{x}+y$ with $y(\mathfrak{h})=0$. Then $\mathfrak{h}^{x}=\left(\mathfrak{h}^{\bar{x}}\right)^{y}$. Our goal is to show that the third condition is also satisfied on a big open subset.

Let $\mathfrak{h}_{\text {sreg }}^{*} \subset \mathfrak{h}_{\text {reg }}^{*}$ be the subset of regular semisimple elements. If $\bar{x} \in \mathfrak{h}_{\text {sreg, }}^{*}$ then the stabiliser $H^{\bar{x}}$ is a torus. Since the action of $H^{\bar{x}}$ on $\operatorname{Ann}(\mathfrak{h}) \subset \mathfrak{g}^{*}$ is self-dual, $\left(\mathfrak{h}^{\bar{x}}\right)^{y}=0$ on a big open subset of $\operatorname{Ann}(\mathfrak{h})$. Assume that $D \subset \mathfrak{g}^{*}$ is an irreducible divisor such that $\mathfrak{h}^{x} \neq 0$ for each $x \in D$. Choose an $H$-stable decomposition $\mathfrak{g}^{*}=\mathfrak{h}^{*} \oplus \operatorname{Ann}(\mathfrak{h})$. Let $p_{1}$ and $p_{2}$ be the projections on the first and the second summands, respectively. The above argument shows that $p_{1}(D)$ is contained in $\mathfrak{h}^{*} \backslash \mathfrak{h}_{\text {sreg }}^{*}$ and hence necessary $\overline{p_{2}(D)}=\operatorname{Ann}(\mathfrak{h})$. Now let $y \in \operatorname{Ann}(\mathfrak{h})$ be a generic point. Then $H^{y}$ is a reductive subgroup of $H$. Arguing by induction on $\operatorname{dim} \mathfrak{g}$ we show that $\left(\mathfrak{h}^{y}\right)^{x^{\prime}}=0$ for all $x^{\prime}$ from a big open subset of $\mathfrak{h}^{*}$. Hence there is no $D$ as above.

Taking the intersection of three big open subsets, we conclude that the differentials $d c_{1}, \ldots, d c_{r}$ are linearly independent on a big open subset. Since each $c_{i}$ is homogeneous, [PPY, Thm 1.1] applies and guarantees that $\mathcal{C}_{1}$ is algebraically closed.

Theorem 3.6 (cf. [Kn90, Satz 2.3]). The conditions (PCm), (Cois), and (DCn) are equivalent.

Proof. For any closed orbit $G x \subset \mathfrak{g}^{*}$, generic $H$-orbits in $G x$ are closed as well [L72]. Hence they are separated by regular $H$-invariants and $\mathbb{k}(G x)^{H}$ is the quotient field of $\mathbb{k}[G x]^{H}$. As $H$ is reductive, $\mathbb{k}[G x]^{H}$ is the restriction of $\mathbb{k}\left[\mathfrak{g}^{*}\right]^{H}$ to $G x$. Thus, $(\mathrm{PCm}) \Rightarrow$ (Cois).

Since $\mathcal{S}(\mathfrak{g})^{\mathfrak{g}}$ is the Poisson centre of $\mathcal{S}(\mathfrak{g})$ and $\mathcal{S}(\mathfrak{h})^{\mathfrak{h}}$ is Poisson-commutative, we have $(\mathrm{DCn}) \Rightarrow(\mathrm{PCm})$.

It remains to show that (Cois) $\Rightarrow$ (DCn). Suppose that (Cois) holds. One of the equivalent interpretations, see (3.5), implies that tr.deg $\mathbb{k}(G x)^{H} \leqslant \mathrm{rkh}$ for each $G x \subset \mathfrak{g}^{*}$. Thereby $\operatorname{tr} \cdot \operatorname{deg} \mathcal{S}(\mathfrak{g})^{\mathfrak{h}} \leqslant \mathrm{rk} \mathfrak{g}+\mathrm{rk} \mathfrak{h}$. We may safely assume that $\mathfrak{h}$ contains no proper ideals of $\mathfrak{g}$. By [Kn90, Satz 2.1], tr.deg $\mathcal{C}_{1}=r \mathfrak{r} \mathfrak{g}+\mathrm{rk} \mathfrak{h}$ for $\mathcal{C}_{1}$ as in Lemma 3.5. Clearly $\mathcal{C}_{1} \subset \mathcal{S}(\mathfrak{g})^{\mathfrak{h}}$ is an algebraic extension. Since $\mathcal{C}_{1}$ is algebraically closed by Lemma 3.5 , we have $\mathcal{C}_{1}=\mathcal{S}(\mathfrak{g})^{\mathfrak{h}}$ and (DCn) holds.

3.3. Cotangent bundles and Richardson orbits. There are similar results for nilpotent orbits, where a different kind of invariant theory is involved. 
Let now $H \subset G$ be an arbitrary reductive subgroup of a reductive group $G$. Take a parabolic $P \subset G$. Then the action of $H$ on $G / P$ is spherical if and only if the action of $H$ on $T^{*}(G / P)$ is coisotropic, see [Kn90', Satz 7.1] and also [Vi01, Chapter 2, §3]. The image of the moment map

$$
\mu: T^{*}(G / P) \rightarrow \mathfrak{g}^{*}
$$

is isomorphic to $G \mathfrak{u}$, where $\mathfrak{u} \subset \mathfrak{p}=$ Lie $P$ is the nilpotent radical of $\mathfrak{p}$. Let $e \in \mathfrak{u}$ be a Richardson element, which means that $\mathcal{O}=G e$ is dense in $G \mathfrak{u}$. Comparing the symplectic structures on $T^{*}(G / P)$ and on $\mathcal{O}$, one obtains the following result.

Theorem 3.7 ([AP14, Thm 2.6]). The action of $H$ on $\mathcal{O}$ is coisotropic if and only if $G / P$ is a spherical $H$-variety.

For a strong Gelfand pair $(G, H)$, this implies that the $H$-action on any Richardson $G$ orbit is coisotropic. Since every nilpotent orbit in $\mathfrak{g l}_{n}$ is Richardson,

$$
\text { the } \mathrm{GL}_{n-1}(\mathbb{k}) \text {-action on any nilpotent adjoint orbit of } \mathrm{GL}_{n}(\mathbb{k}) \text { is coisotropic. }
$$

Coisotropic actions of subgroups $Q \subset G$ on adjoint orbits of a semisimple group $G$ have also been studied in [Z09].

\section{THE POLYNOMIAL GELFAND-TSETLIN INTEGRABLE SYSTEM IN TYPE A}

In this section, $\mathfrak{g}=\mathfrak{g l}_{n}=\mathfrak{g l}_{n}(\mathbb{k})$. Let $\left\{E_{i j}\right\}_{i, j=1}^{n} \in \mathfrak{g l}_{n}$ be the matrix units. Fix the chain of subalgebras

$$
\mathfrak{g l}_{n} \supset \mathfrak{g l}_{n-1} \supset \ldots \supset \mathfrak{g l}_{2} \supset \mathfrak{g l}_{1},
$$

where $\mathfrak{g l}_{n-k}=\left\langle E_{i j} \mid i, j>k\right\rangle_{\mathfrak{k}}$. In other words, let us fix a basis $\left\{v_{1}, \ldots, v_{n}\right\}$ for $V_{n}=\mathbb{k}^{n}$ and set $V_{j}=\left\langle v_{n-j+1}, \ldots, v_{n}\right\rangle_{\mathbb{k}}$. Then $V_{n} \supset \cdots \supset V_{1}$ is a full flag and $\mathfrak{g l}_{j}=\mathfrak{g l}\left(V_{j}\right)$ for all $j$. For any matrix $A \in \mathfrak{g l}_{n}$, let $A_{m}$ denote the south-east corner of $A$ of size $n-m$, i.e., $A_{m} \in \mathfrak{g l}_{n-m}$. For each $m \in\{0,1, \ldots, n-1\}$, let $\left\{\Delta_{k}^{[m]} \mid 1 \leqslant k \leqslant n-m\right\}$ be the coefficients of the characteristic polynomials of $A_{m}$. Here $\Delta_{k}^{[m]} \in \mathcal{S}^{k}\left(\mathfrak{g l}_{n-m}\right) \subset \mathcal{S}^{k}\left(\mathfrak{g l}_{n}\right)$, and we also write $\Delta_{k}=\Delta_{k}^{[0]}$.

The Gelfand-Tsetlin $(=G T)$ subalgebra $\mathcal{C} \subset \mathcal{S}\left(\mathfrak{g l}_{n}\right)$ is generated by

$$
\Delta_{1}, \ldots, \Delta_{n}, \Delta_{1}^{[1]}, \ldots, \Delta_{n-1}^{[1]}, \Delta_{1}^{[2]}, \ldots, \Delta_{k}^{[m]}, \ldots, \Delta_{1}^{[n-1]} .
$$

Note that $\mathcal{C}=\operatorname{gr}(\widetilde{\mathcal{C}})$, where $\widetilde{\mathcal{C}} \subset \mathcal{U}\left(\mathfrak{g l}_{n}\right)$ is the commutative subalgebra defined and studied by Gelfand and Tsetlin [GT50]. Therefore, these generators are algebraically independent, tr.deg $\mathcal{C}=\boldsymbol{b}\left(\mathfrak{g l}_{n}\right)$, and $\{\mathcal{C}, \mathcal{C}\}=0$.

By [T02], $\mathcal{C}$ is a maximal Poisson-commutative subalgebra of $\mathcal{S}\left(\mathfrak{g l}_{n}\right)$. The same result is independently obtained in [KW06, Thm 3.25]. Kostant and Wallach also prove that $\mathcal{C}$ is 
complete on every regular orbit, see Theorem 3.36 in loc. cit.

We prove below that $\mathcal{C}$ is complete on every (co)adjoint orbit.

Definition 3. A matrix $A \in \mathfrak{g l}_{n}$ is said to be

(i) strongly regular, if $\operatorname{dim} d_{A} \mathrm{e}=\boldsymbol{b}\left(\mathfrak{g l}_{n}\right)$;

(ii) strongly nilpotent, if $\Delta_{k}^{[m]}\left(A_{m}\right)=\Delta_{k}^{[m]}(A)=0$ for $0 \leqslant m \leqslant n-1$ and $1 \leqslant k \leqslant n-m$.

Theorem 4.1. Any nilpotent orbit $\mathcal{O} \subset \mathfrak{g}^{*}$ contains a strongly nilpotent element $e \in \mathcal{O}$ such that

$$
\operatorname{dim} d_{e} \mathcal{e}=n+\frac{1}{2} \operatorname{dim} \mathcal{O} \text { and } \operatorname{dim}\left(d_{e} \mathcal{C} \cap \mathfrak{g}^{e}\right)=n .
$$

In particular, $\mathrm{C}$ is complete on $\mathcal{O}$.

Proof. As above, for $e \in \mathfrak{g l}_{n} \simeq \mathfrak{g l}_{n}^{*}$, let $e_{m} \in \mathfrak{g l}_{n-m}$ denote the corresponding south-east corner, where $0 \leqslant m<n$. In particular, $e_{0}=e$ and $e_{n-1} \in \mathfrak{g l}_{1}$. If all $\left\{e_{m}\right\}$ are nilpotent, then $d_{e} \Delta_{k}^{[m]}=\left(e_{m}\right)^{k-1}$ as a matrix.

Let $\mathcal{O}=\mathcal{O}(\boldsymbol{r})$, where $\boldsymbol{r}=\left(r_{1}, r_{2}, \ldots, r_{t}\right)$ is the corresponding partition of $n$. If $t=1$, i.e., $r_{1}=n$, then $\mathcal{O}=\mathcal{O}(n)$ is regular and a Jordan normal form adapted to the chain (4.1) provides a strongly nilpotent element in $\mathcal{O}$. Namely, take a basis $\left\{v_{1}, \ldots, v_{n}\right\}$ for $\mathbb{k}^{n}$ as above and set $e v_{j}=v_{j+1}$ for all $j$. (Here and below we assume that $v_{j}=0$ for $j>n$.) In this case, $d_{e} \mathfrak{C}=\mathfrak{b}$, the unique Borel subalgebra containing $e$, and the assertions are clear. Therefore, we always assume below that $t \geqslant 2$, i.e., $r_{2}>0$.

Let $e^{\prime} \in \mathfrak{g l}_{n-1}$ be a nilpotent element defined by the partition $\boldsymbol{r}_{1}=\left(r_{1}+r_{2}-1, r_{3}, \ldots, r_{t}\right)$. As the next step we will construct a representative $e \in \mathcal{O}$ such that $e^{\prime}=e_{1}$. Our construction will not affect the Jordan blocks for $r_{3}, \ldots, r_{m}$. Set $c=r_{1}+r_{2}$.

Let $\left\{v_{2}, \ldots, v_{c}\right\}$ be a Jordan basis for the first block of $e^{\prime}$, i.e., $e^{\prime} v_{j}=v_{j+1}$ for $2 \leqslant j \leqslant c-1$ and $e^{\prime} v_{c}=0$. Define $e \in \mathfrak{g l}_{n}$ as follows:

$$
e v_{1}=-v_{r_{1}+2}, e v_{r_{1}}=v_{r_{1}+1}+v_{1}, e v_{c}=0 \text {, and } e v_{j}=e^{\prime} v_{j}=v_{j+1} \text { for } j \neq 1, r_{1}, c .
$$

Then $\left\{v_{2}, \ldots, v_{r_{1}}, v_{r_{1}+1}+v_{1}\right\}$ is a Jordan basis for the block of size $r_{1}$ for $e$ and if $r_{2} \geqslant 2$, then $\left\{\frac{1}{2}\left(v_{r_{1}+1}-v_{1}\right), v_{r_{1}+2}, \ldots, v_{c}\right\}$ is a Jordan basis for the block of size $r_{2}$ for $e$. For $r_{2}=1$, the second block consists of $v_{r_{1}+1}-v_{1}$ or just $v_{1}$.

In Example 2.10, we have constructed the colour pattern associated with $\mathcal{O}(\boldsymbol{r})$. For further considerations, replace each $\partial_{a}^{m} H_{k}$ in that pattern with $\Delta_{k-m}^{[m]}$.

In order to prove the theorem, we argue by induction on $n$. The case $n=1$ is void. By the inductive hypothesis, both equalities of the theorem hold for $e_{1}$. Observe that the colour pattern associated with $\mathcal{O}\left(\boldsymbol{r}_{1}\right)$ can be obtained from that of $\mathcal{O}(\boldsymbol{r})$ in two steps. First, we cut the top row, thus, producing a wrong pattern, as the last $r_{1}-1$ columns begin with a green box. Second, these boxes are repainted red. The figure bellow illustrates the passage from $\mathcal{O}(3,2,1)$ to $\mathcal{O}(4,1)$. 

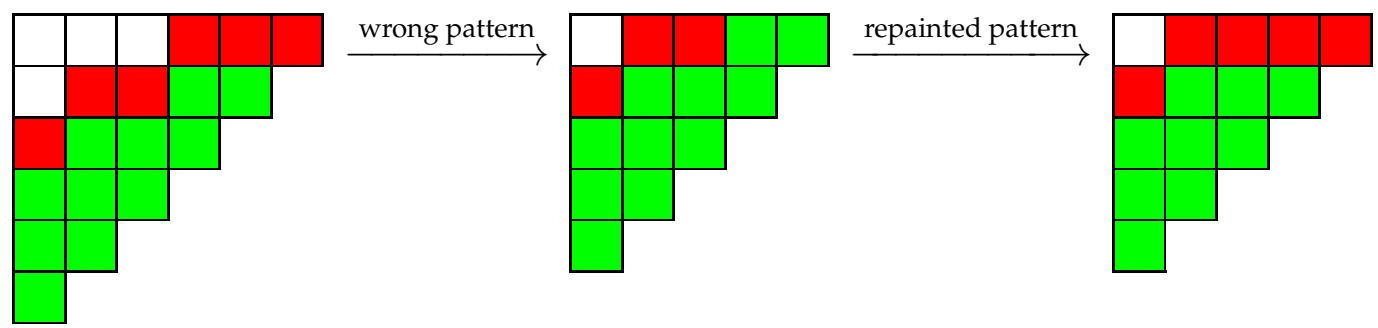

Recall from Proposition 2.9 and Example 2.10 that in the pattern related to $\mathcal{O}$ the number of coloured boxes equals $n+\frac{1}{2} \operatorname{dim} \mathcal{O}$ and the number of green boxes equals $\frac{1}{2} \operatorname{dim} \mathcal{O}$.

By the inductive hypothesis,

$$
\operatorname{dim}\left\langle\boldsymbol{d}_{e_{1}} \Delta_{k}^{[m]} \mid m \geqslant 1\right\rangle_{\mathbb{k}}=(n-1)+\frac{1}{2} \operatorname{dim} \mathcal{O}\left(\boldsymbol{r}_{1}\right)=\left(n+\frac{1}{2} \operatorname{dim} \mathcal{O}\right)-r_{1} .
$$

Observe that $d_{e} \Delta_{k}^{[m]}=d_{e_{1}} \Delta_{k}^{[m]} \in \mathfrak{g l}_{n-1}$ for $m \geqslant 1$. As can be easily seen, the matrices $e^{k}=d_{e} \Delta_{k+1}$ with $0 \leqslant k<r_{1}$ are linearly independent. Furthermore, $e^{k}=0$ for $k \geqslant r_{1}$. In order to show that $d_{e} \mathcal{C}$ has the required dimension, it is enough to prove that

$$
\left\langle e^{0}, \ldots, e^{r_{1}-1}\right\rangle_{\mathbb{k}} \cap \mathfrak{g l}_{n-1}=0 .
$$

For $0<k<r_{1}$, we have

$$
e^{k} v_{r_{1}-k+1}=e v_{r_{1}}=v_{r_{1}+1}+v_{1} \text { and } e^{k} v_{s} \in\left\langle v_{2}, \ldots, v_{c}\right\rangle_{\mathbb{k}} \text { if } s \neq r_{1}-k+1 \text {. }
$$

Also $e^{0} v_{k}=v_{k}$ for $2 \leqslant k \leqslant c$. Since the vectors $v_{2}, \ldots, v_{r_{1}}$ are linearly independent,

$$
\left\langle e^{0}, \ldots, e^{r_{1}-1}\right\rangle_{\mathbb{k}} \cap \mathfrak{g l}_{n-1} \subset\left(\mathbb{k} e^{0} \cap \mathfrak{g l}_{n-1}\right) .
$$

Clearly, $e^{0} \notin \mathfrak{g l}_{n-1}$. Therefore $\operatorname{dim} \boldsymbol{d}_{e} \mathrm{e}=n+\frac{1}{2} \operatorname{dim} \mathcal{O}$.

The behaviour of $\mathcal{C}$ on $\mathcal{O}$ is a more delicate question. Recall that $\mathfrak{g}^{e}$ is the kernel of the canonical projection $T_{e}^{*} \mathfrak{g}^{*} \rightarrow T_{e}^{*} \mathcal{O}$. Furthermore, we need the following obvious observations: $d_{e} \Delta_{k} \in \mathfrak{g}^{e}$ for each $k$ and $\operatorname{dim} \mathcal{O}-\operatorname{dim} \mathcal{O}\left(\boldsymbol{r}_{1}\right)=2\left(r_{1}-1\right)$. By the inductive hypothesis, the images of $d_{e} \Delta_{k}^{[m]}$ with $m>1$ under the projection

$$
\mathfrak{g l}_{n-1} \rightarrow \mathfrak{g l}_{n-1} /\left(\mathfrak{g l}_{n-1}\right)^{e_{1}}
$$

span a subspace of dimension $\frac{1}{2} \operatorname{dim} \mathcal{O}\left(\boldsymbol{r}_{1}\right)$.

Consider now the green elements $\Delta_{k}^{[1]}$. Here $1 \leqslant k \leqslant r_{1}-1$ and $d_{e} \Delta_{k}^{[1]}=d_{e_{1}} \Delta_{k}^{[1]}=e_{1}^{k-1}$. Clearly, $e_{1}^{k} \in\left(\mathfrak{g l}_{n-1}\right)^{e_{1}}$ for each $k$. In order to finish the proof it suffices to show that the differentials $e_{1}^{k}$ with $0 \leqslant 0 \leqslant r_{1}-2$ remain linearly independent on $T_{e} \mathcal{O}=\operatorname{ad}^{*}(\mathfrak{g}) e$.

Let $y \in\left(\mathfrak{g l}_{n}\right)^{e}$. Using elementary properties of centralisers [Y09, Sect. 1], one readily sees that $v_{r_{1}+1}-v_{1}$ does not lie in

$$
R(y)=y\left\langle v_{3}, \ldots, v_{r_{1}}, v_{r_{1}+1}+v_{1}\right\rangle_{\mathbb{k}}+\left\langle v_{3}, \ldots, v_{r_{1}}, v_{r_{1}+1}+v_{1}, v_{r_{1}+2}, \ldots, v_{c}\right\rangle_{\mathbb{k}} .
$$


Assume that there is a non-trivial linear combination $y=\beta_{0} e_{1}^{0}+\ldots+\beta_{r_{1}-2} e_{1}^{r_{1}-2}$ such that $y \in \mathfrak{g}^{e}$. Take the smallest $k \geqslant 0$ with $\beta_{k} \neq 0$. Then $y v_{r_{1}+1-k} \in \beta_{k} v_{r_{1}+1}+\left\langle v_{r_{1}+2}, \ldots, v_{c}\right\rangle_{\mathbb{k}}$. Here $r_{1}+1-k \geqslant 3$ and $v_{r_{1}+1}-v_{1} \in R(y)$, a contradiction!

Remark 4.2. (i) The strategy used in the proof of Theorem 4.1 is suggested by a connection between MF- and GT-subalgebras. Namely, by a result of Vinberg, $\mathcal{C}$ can be realised as a limit of MF-subalgebras. That is, if

$$
a(t)=E_{11}+t E_{22}+\ldots+t^{n-1} E_{n n},
$$

then $\lim _{t \rightarrow 0} \mathcal{F}_{a(t)}=\mathcal{C}$ for the chain as above, see [Vi91, 6.4]. Even more explicitly, in $\mathbb{P}\left(\mathcal{S}^{k-m}\left(\mathfrak{g l}_{n}\right)\right)$, we have $\lim _{t \rightarrow 0}\left\langle\partial_{a(t)}^{m} \Delta_{k}\right\rangle=\left\langle\Delta_{k-m}^{[m]}\right\rangle$, cf. [MY17, Ex. 5.5].

The properties of $\mathcal{F}_{a(t)}$ and its restriction to $\mathcal{O}$, see Proposition 2.9 and Example 2.10, suggest how to construct bases for $d_{e} \mathcal{C}$ and $d_{e}\left(\left.\mathcal{C}\right|_{\mathcal{O}}\right)=\left(d_{e} \mathcal{C}\right) /\left(\left(\mathfrak{g l}_{n}\right)^{e} \cap d_{e} \mathcal{C}\right)$. Indeed, as we have seen in the proof of Theorem 4.1, the differentials of the coloured elements $\Delta_{k}^{[m]}$ form a basis of $d_{e} \mathcal{C}$. By the definition of a colour pattern, $d_{e}\left(\partial_{a(t)}^{k} H_{i}\right) \in\left(\mathfrak{g l}_{n}\right)^{e}$ for the red elements $\partial_{a(t)}^{k} H_{i}$. From this one can deduce that the differentials $d_{e} \Delta_{k}^{[m]}$ with red $\Delta_{k}^{[m]}$ form a basis of $d_{e} \mathcal{C} \cap\left(\mathfrak{g l}_{n}\right)^{e}$. The uncoloured elements $\Delta_{k}^{[m]}$ restrict to zero on $\mathcal{O}$.

(ii) Let $\mathcal{A}=\lim _{t \rightarrow 0} \mathcal{F}_{a(t)}$ with $a(t) \in \mathfrak{t}$ be a limit in the sense of [Vi91, 6.4]. According to [T02], $\operatorname{dim} d_{x} \mathcal{A}=\boldsymbol{b}(\mathfrak{g})$ for each $x \in \mathcal{K}$, where $\mathcal{K}$ is the Kostatn section as in the proof of Theorem 2.4. Therefore $\mathcal{A}$ is complete on any regular orbit, cf. Lemma 1.1.

Theorem 4.3. The GT-subalgebra $\mathrm{C}$ is complete on every adjoint orbit of $G=\mathrm{GL}_{n}$.

Proof. For a nilpotent orbit $G e$, the result follows from Theorem 4.1. Proposition 2.5 immediately extends it to all orbits.

Theorem 4.4. The action of $\mathrm{GL}_{n-1}$ on each adjoint orbit $\mathrm{GL}_{n} x \subset \mathfrak{g l}_{n}$ is coisotropic.

Proof. By Theorem 4.3, $\mathrm{C}$ is complete on every adjoint orbit. More precisely, since $\Delta_{1}, \ldots, \Delta_{n}$ are constant on the orbits, the proper subalgebra $\mathcal{C} \cap \mathcal{S}\left(\mathfrak{g l}_{n-1}\right)$ is complete on every orbit $\mathrm{GL}_{n} x \subset \mathfrak{g l}_{n}$. This family consists of Noether integrals. The discussion in Section 3.1 and, in particular, assertion (NF) show that the action of $\mathrm{GL}_{n-1}$ on $\mathrm{GL}_{n} x$ is coisotropic.

Remark 4.5. (i) Note that Theorem 4.3 provides a new unusual proof of Elashvili's conjecture in type A. The argument goes as follows. Take $x \in \mathfrak{g l}_{n}^{*}$ such that $\left(\mathfrak{g l}_{n}\right)^{x} \neq \mathfrak{g l}_{n}$. Since $\mathcal{C}$ is complete on $\mathrm{GL}_{n} x$ and $\mathcal{C}=\lim _{t \rightarrow 0} \mathcal{F}_{a(t)}$, the MF subalgebra $\mathcal{F}_{a(t)}$ is complete on $\mathrm{GL}_{n} x$ for at least one $t \in \mathbb{k}^{\times}$. Then according to Lemma 2.1, ind $\left(\mathfrak{g l}_{n}\right)^{x}=\mathrm{rkgl}_{n}$.

(ii) Theorem 4.3 has a different, more sophisticated and inductive line of argument that does not involve the direct calculation of Theorem 4.1. Suppose that the statement holds for $\mathrm{GL}_{n-1}$. Take a nilpotent orbit $G e \subset \mathfrak{g}^{*}$. The Gelfand-Tsetlin subalgebra of $\mathcal{S}\left(\mathfrak{g l}_{n-1}\right)$ 
separates generic $\mathrm{GL}_{n-1}$-orbits on the image $\mu(G e) \subset \mathfrak{g l}_{n-1}^{*}$ and is complete on each orbit of $\mathrm{GL}_{n-1}$. It can be deduced from (3.6) that the Gelfand-Tsetlin subalgebra of $\mathcal{S}\left(\mathfrak{g l}_{n-1}\right)$ is complete on $G e$. Hence $\mathcal{C}$ is complete on Ge. By Proposition 2.5, $\mathcal{C}$ is complete on every adjoint orbit.

4.1. $\lambda$-systems. In their approach to GT integrable systems, Guillemin and Sternberg prefer to deal with eigenvalues of Hermitian matrices (i.e., piecewise smooth functions) [GS83]. Take the compact form $\mathfrak{k}=\mathfrak{u}_{n}$ and identify $\mathfrak{k}^{*}$ with $i_{\mathfrak{u}_{n}}$. Now the eigenvalues $\left\{\lambda_{k}\right\}$ of $A \in \mathfrak{k}^{*}$ are real numbers. Let $\boldsymbol{\lambda}_{k}$ with $1 \leqslant k \leqslant n$ be the corresponding functions on $\mathfrak{k}^{*}$, i.e., $\boldsymbol{\lambda}_{k}(A)=\lambda_{k}$, and likewise for $\boldsymbol{\lambda}_{k}^{[m]}$. The completely integrable system on $K A \subset \mathfrak{k}^{*}$ is given by the restrictions of $\left\{\boldsymbol{\lambda}_{k}^{[m]} \mid 1 \leqslant m<n \& 1 \leqslant k \leqslant n-m\right\}$. We call it the $\boldsymbol{\lambda}$-system.

There is an obvious connection between $\mathcal{C}$ and the $\lambda$-system. Let $\sigma_{k}$ be the $k$-th elementary symmetric polynomial. If one defines $\boldsymbol{\lambda}_{k}$ over $\mathbb{k}$ or considers $\Delta_{k}$ as real valued functions on $\mathfrak{k}^{*}$, then $\Delta_{k}=\sigma_{k}\left(\boldsymbol{\lambda}_{1}, \ldots, \boldsymbol{\lambda}_{n}\right)$. Take $A \in \mathfrak{u}_{n}^{*} \subset \mathfrak{g l}_{n}(\mathbb{C})^{*}$. Using a standard argument, one proves that

the $\lambda$-system is complete on $\mathrm{U}_{n} A \Longleftrightarrow \mathcal{C}$ is complete on $\mathrm{GL}_{n}(\mathbb{C}) A$.

Moreover, we see that there is a connection between the $\lambda$-system and the colour patterns used in the proof of Theorem 4.1.

Until the end of this section, assume that $\mathbb{k}=\mathbb{C}$ and therefore $\mathrm{GL}_{n}=\mathrm{GL}_{n}(\mathbb{C})$. Let $\mathcal{O}$ be the dense orbit in the associated cone of $\mathrm{GL}_{n} A$. Then for each $m$, the number of elements $\lambda_{k}^{[m]}$ with $1 \leqslant k \leqslant n-m$ that are functionally independent on $\mathrm{U}_{n} A$ is equal to the number of green elements $\Delta_{k}^{[m]}$ in the colour pattern associated with $\mathcal{O}$. This connection explains also the choice of $e^{\prime}$ in the proof of Theorem 4.1.

Let $\lambda_{1} \leqslant \ldots \leqslant \lambda_{n}$ be the eigenvalues of $A$. Let $A_{1} \in \mathfrak{u}_{n-1}^{*}$ denote the restriction of $A$ to $\mathfrak{u}_{n-1}$. If $\mu_{1} \leqslant \ldots \leqslant \mu_{n-1}$ are the eigenvalues of $A_{1}$, then $\lambda_{i} \leqslant \mu_{i} \leqslant \lambda_{i+1}$. For a nonregular orbit $\mathrm{U}_{n} A, \lambda_{i+1}=\lambda_{i}$ for some $i$. Therefore, gathering together equal eigenvalues of $A$, we get a partition of $n$ different from $\left(1^{n}\right)$. The parts of the dual partition, say $r_{1} \geqslant \ldots \geqslant r_{t}>0$, are the sizes of the Jordan blocks of $e \in \mathcal{O}$ [K76]. Suppose that $A$ is a generic representative of $\mathrm{U}_{n} A$. The key point in the complete integrability of $\boldsymbol{\lambda}$ on $\mathrm{U}_{n} A$ [GS83] is that the eigenvalues of $A_{1}$ are not equal if they do not have to be. In other words, the associated cone of $\mathrm{GL}_{n-1} A_{1}$ is the closure of $\mathrm{GL}_{n-1} e^{\prime}$, where $e^{\prime}$ is given by the partition $\left(r_{1}+r_{2}-1, r_{3}, \ldots, r_{t}\right)$.

Example 4.6. Let $A \in \mathfrak{u}_{7}^{*}$ have the eigenvalues

$$
\lambda_{1}=\lambda_{2}=\lambda_{3}<\lambda_{4}=\lambda_{5}<\lambda_{6}=\lambda_{7} .
$$

This means that $\mu_{1}=\mu_{2}$, but there are no other necessary equalities among the eigenvalues of $A_{1}$. In terms of partitions, this set of eigenvalues gives rise to the partition $(3,2,2)$, 
with the dual partition $\boldsymbol{r}=(3,3,1)$. Then $\boldsymbol{r}_{1}=(5,1)$, and its dual is $(2,1,1,1,1)$. This last partition describes the coincidence of the eigenvalues of $A_{1}$.

On the orbit $\mathrm{U}_{7} A$, we have $\mu_{1}=\mu_{2}=\lambda_{1}$ as well as $\mu_{4}=\lambda_{4}$ and $\mu_{6}=\lambda_{6}$. Among the function $\lambda_{k}^{[1]}$, only two, namely $\lambda_{3}^{[1]}$ and $\lambda_{5}^{[1]}$, are functionally independent. According to the colour pattern used in the proof of Theorem 4.1, the images of the differentials $d_{A} \lambda_{k}^{[1]}$ with $1 \leqslant k \leqslant 6$ span a subspace of dimension 2 in the quotient of $T_{A}^{*} \mathfrak{u}_{7}^{*}$ by $\mathfrak{u}_{7}^{A}$.

\section{CORANK ON CLOSURES OF SHEETS AND THE ORTHOGONAL CASE}

Let $(M, \omega)$ and $Q$ be as in Section 3.1. Set $U:=\left\{y \in M \mid \operatorname{dim}(\mathfrak{q} y)=\max _{x \in M} \operatorname{dim}(\mathfrak{q} x)\right\}$.

Definition 4. The defect of the $Q$-action on $M$ is

$$
\operatorname{def}(M)=\operatorname{def}_{Q}(M)=\min _{y \in U} \operatorname{dim}\left(\mathfrak{q} y \cap(\mathfrak{q} y)^{\perp}\right) ;
$$

and the corank of the $Q$-action is cork $(M)=\operatorname{cork}_{Q}(M):=\max _{y \in U} \mathrm{rk}\left(\left.\omega_{y}\right|_{(\mathfrak{q} y)^{\perp}}\right)$.

If $x \in U$, then $\operatorname{cork}(M)=\operatorname{dim} M-\operatorname{dim}(\mathfrak{q} x)-\operatorname{def}(M)$. We omit the indication of $Q$ if it is clear from the context. The coisotropic actions are of corank zero.

From now on, suppose that $M$ is an irreducible algebraic variety defined over $\mathbb{k}$. Then the image $\mu(M) \subset \mathfrak{q}^{*}$ is a $Q$-stable subset, which is dense in its closure. Moreover, $\overline{\mu(M)}$ is irreducible. For an irreducible $Q$-stable closed subset $Y \subset \mathfrak{q}^{*}$, set

$$
\boldsymbol{b}(Y)=\operatorname{dim} Y-\frac{1}{2} \max _{y \in Y} \operatorname{dim}(\mathfrak{q} y) .
$$

The transcendence degree of a Poisson-commutative subalgebra of $\mathbb{k}(Y)$ is bounded above by $\boldsymbol{b}(Y)$. Note that $\boldsymbol{b}\left(\mathfrak{q}^{*}\right)=\boldsymbol{b}(\mathfrak{q})$ is just the "magic number". Note also that $\max _{y \in \overline{\mu(M)}} \operatorname{dim}(\mathfrak{q} y)=\max _{y \in \mu(M)} \operatorname{dim}(\mathfrak{q} y)$. Set $\boldsymbol{b}(\mu(M))=\boldsymbol{b}(\overline{\mu(M)})$.

The equality ker $d_{x} \mu=(\mathfrak{q} x)^{\perp}$ that has been discussed in Section 3.1 leads to the following formulas:

$$
\begin{aligned}
& \operatorname{dim} \overline{\mu(M)}=\operatorname{dim}(\mathfrak{q} x) \text { for } x \in U ; \\
& \max _{y \in \mu(M)} \operatorname{dim}(\mathfrak{q} y)=\operatorname{dim}(\mathfrak{q} x)-\operatorname{def}(M) \text { for } x \in U ; \\
& 2 \boldsymbol{b}(\mu(M))+\operatorname{cork}(M)=2 \operatorname{dim}(\mathfrak{q} x)-\operatorname{dim}(\mathfrak{q} x)+\operatorname{def}(M)+\operatorname{cork}(M)=\operatorname{dim} M .
\end{aligned}
$$

By [VY18], for any $Q$-stable irreducible closed subset $Y \subset \mathfrak{q}^{*}$, there is a subalgebra $\mathcal{A} \subset \mathcal{S}(\mathfrak{q})$ such that $\{\mathcal{A}, \mathcal{A}\}$ vanishes on $Y$ and $\operatorname{tr} \cdot \operatorname{deg}\left(\left.\mathcal{A}\right|_{Y}\right)=\boldsymbol{b}(Y)$. If $Y=\overline{\mu(M)}$ and the action of $Q$ on $M$ is coisotropic, then the pull-back $\mu^{*}(\mathcal{A})$ contains a complete family of functions, Noether integrals, on $M$. We only need these statements if $Q$ is reductive, in which case the proof simplifies drastically. 
Lemma 5.1 (cf. [VY18, Sect. 3]). Suppose that $Q$ is reductive. Then there is $a \in \mathfrak{q}^{*}$ such that $\operatorname{tr} \cdot \operatorname{deg}\left(\left.\mathcal{F}_{a}\right|_{Y}\right)=\boldsymbol{b}(Y)$ for the MF-subalgebra $\mathcal{F}_{a}$ associated with a.

Proof. Since $Y \subset \mathfrak{q}^{*}$, each fibre of the quotient map $Y \rightarrow Y / / Q$ contains an open orbit. Therefore $\operatorname{dim} Y / / Q=\operatorname{dim} Y-r$, where $r=\max _{y \in Y} \operatorname{dim}(\mathfrak{q} y)$. Hence also $\operatorname{dim} d_{y}\left(\mathcal{S}(\mathfrak{q})^{\mathfrak{q}}\right)=$ $\operatorname{dim} Y-r$ for generic $y \in Y$. Fix one $y \in Y$ having this property. There is $a \in \mathfrak{q}^{*}$ such that $\mathcal{F}_{a}$ is complete on $Q y$, see [B91] and Section 2. Since $\mathcal{S}(\mathfrak{q})^{\mathfrak{q}} \subset \mathcal{F}_{a}$, we conclude that $\operatorname{tr} \cdot \operatorname{deg}\left(\left.\mathcal{F}_{a}\right|_{Y}\right)=r+\frac{1}{2} \operatorname{dim}(Q y)=\boldsymbol{b}(Y)$.

5.1. Numerical invariants of sheets. Let $H$ be an arbitrary reductive subgroup of a connected reductive group $G$. Let $S \subset \mathfrak{g}$ be a $G$-sheet and $G e$ the unique nilpotent orbit in $S$, see [BK79, Sect. 5.8, Kor.(a)]. For any coadjoint orbit $G x \subset \mathfrak{g}^{*}$, the moment map w.r.t. $H, \mu: G x \rightarrow \mathfrak{h}^{*}$, is given by the restriction $\mathfrak{g}^{*} \rightarrow \mathfrak{h}^{*}$ of linear functions. The dual map (co-morphism) $\mu^{*}$ is the canonical inclusion $\mathcal{S}(\mathfrak{h}) \subset \mathcal{S}(\mathfrak{g})$.

Lemma 5.2. For any $G$-orbit $\mathcal{O} \subset S$, one has $\operatorname{cork}_{H}(\mathcal{O}) \leqslant \operatorname{cork}_{H}(G e)$.

Proof. Set $Y=\overline{\mu(G e)}$. This is an $H$-stable irreducible closed subset of $\mathfrak{h}^{*}$. By Lemma 5.1, there is $a \in \mathfrak{h}^{*}$ such that $\operatorname{tr} \cdot \operatorname{deg}\left(\left.\mathcal{F}_{a}\right|_{Y}\right)=\boldsymbol{b}(Y)$ for the MF-subalgebra $\mathcal{F}_{a} \subset \mathcal{S}(\mathfrak{h})$. Note that $\mathcal{F}_{a}$ is a homogeneous Poisson-commutative subalgebra of $\mathcal{S}(\mathfrak{g})$. For each $G x \subset S$, the orbit $G e$ is dense in the associated cone of $G x$. Making use of (2.5), we write

$$
\boldsymbol{b}(Y)=\operatorname{tr} \cdot \operatorname{deg}\left(\left.\mathcal{F}_{a}\right|_{G e}\right) \leqslant \operatorname{tr} \cdot \operatorname{deg}\left(\left.\mathcal{F}_{a}\right|_{G x}\right) \leqslant \boldsymbol{b}(\mu(G x)) .
$$

By (5.4), we have $\operatorname{cork}_{H}(M)=\operatorname{dim} M-2 \boldsymbol{b}(\mu(M))$. Since $\operatorname{dim}(G x)=\operatorname{dim}(G e)$, the result follows.

Lemma 5.3. For any $G$-orbit $\mathcal{O} \subset \mathfrak{g}^{*}$, the corank $\operatorname{cork}_{H}(\mathcal{O})$ is equal to the rank of $\hat{x}$ on $d_{x}\left(\mathcal{S}(\mathfrak{g})^{H}\right)$ for a generic $x \in \mathcal{O}$.

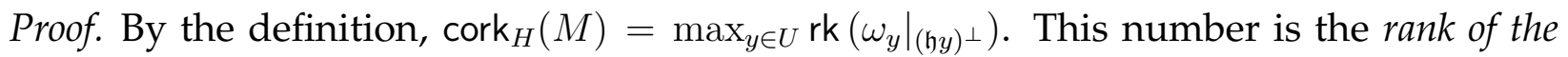
Poisson bracket on $\mathbb{k}(M)^{H}$. Suppose that $F_{1}, \ldots, F_{k} \in \mathbb{k}(\mathcal{O})^{H}$ are algebraically independent and $k=\operatorname{tr} \cdot \operatorname{deg} \mathbb{k}(\mathcal{O})^{H}$. Whenever all $d_{y} F_{i}$ are defined for $y \in \mathcal{O}$, set

$$
V(y)=\left\langle d_{y} F_{i} \mid 1 \leqslant i \leqslant k\right\rangle_{\mathbb{k}} .
$$

Then $\operatorname{cork}_{H}(\mathcal{O})=\max _{y \in \mathcal{O}}$ rk $\left(\left.\hat{y}\right|_{V(y)}\right)$. In [AP14, Prop. 2.9], it is explained how to deduce from results of [Lo09] the fact that $\mathbb{k}(\mathcal{O})^{H}=\operatorname{Quot}\left(\mathbb{k}[\mathcal{O}]^{H}\right)$. By [BK79, Lemma 3.7], $\mathbb{k}[\mathcal{O}]$ is an integral extension of $\mathbb{k}[\overline{\mathcal{O}}]$. Hence $\mathbb{k}[\mathcal{O}]^{H}$ is an algebraic extension of $\mathbb{k}[\overline{\mathcal{O}}]^{H}$. Summing up, tr.deg $\mathbb{k}(\mathcal{O})^{H}=\operatorname{tr} \cdot \operatorname{deg} \mathbb{k}[\overline{\mathcal{O}}]^{H}$.

Since $H$ is reductive, $\mathbb{k}[\overline{\mathcal{O}}]^{H}$ is the image of $\mathbb{k}\left[\mathfrak{g}^{*}\right]^{H}$ under the restriction to $\overline{\mathcal{O}}$. Hence $V(y)=d_{y}\left(\mathcal{S}(\mathfrak{g})^{H}\right) / \mathfrak{g}^{y}$ on a non-empty open subset of $\mathcal{O}$. Since $\mathfrak{g}^{y}$ is the kernel of $\hat{y}$, the result follows. 
Theorem 5.4. Let $S \subset \mathfrak{g}^{*}$ be a sheet.

(i) The corank of the $H$-action on $G$-orbits does not change along $S$;

(ii) if a $G$-orbit $\mathcal{O}$ lies in $\bar{S}$, then $\operatorname{cork}_{H}(\mathcal{O}) \leqslant \operatorname{cork}_{H}(G x)$ with $x \in S$.

Proof. Lemma 5.3 readily implies that there is a dense subset of $S$ such that $\operatorname{cork}(G x)=r$ for each orbit $G x$ in this subset and $\operatorname{cork}(\mathcal{O}) \leqslant r$ for each orbit $\mathcal{O} \subset \bar{S}$.

Making use of Lemma 5.2, we show that $r \leqslant \operatorname{cork}(G e) \leqslant r$. Hence cork $(G e)=r$. Finally suppose that $G y \subset S$ is not nilpotent. Then $G e \subset \overline{\mathbb{k}^{\times} G y}$ and in view of Lemma 5.3 $\operatorname{cork}(G y) \geqslant \operatorname{cork}(G e)=r$. At the same time $\operatorname{cork}(G y) \leqslant r$. This finishes the proof.

There are many other characteristics of $H$-actions that do not change along a sheet.

Theorem 5.5. Let $S \subset \mathfrak{g}$ be a sheet with unique nilpotent orbit $G e$. Take $G x \subset S$. Then

(1) $\operatorname{tr} \cdot \operatorname{deg} \mathbb{k}[G x]^{H}=\operatorname{tr} \cdot \operatorname{deg} \mathbb{k}[G e]^{H}$;

(2) $\max _{x^{\prime} \in G x} \operatorname{dim}\left(H x^{\prime}\right)=\max _{e^{\prime} \in G e} \operatorname{dim}\left(H e^{\prime}\right)$;

(3) $\operatorname{dim} \mu(G x)=\operatorname{dim} \mu(G e)$;

(4) $\operatorname{def}(G x)=\operatorname{def}(G e)$;

(5) $\max _{\xi \in \mu(G x)} \operatorname{dim}(H \xi)=\max _{\eta \in \mu(G e)} \operatorname{dim}(H \eta)$.

Proof. We can safely assume that $x \notin G e$ and therefore is not nilpotent. Let $F \in \mathbb{k}\left[\mathfrak{g}^{*}\right]$ be a homogenous $G$-invariant that is non-zero on $G x$. Then $\overline{G e} / / H$ is defined as the zero set of $F$ in $\overline{\mathbb{k}^{\times} G x} / / H$. Hence $\operatorname{dim} \overline{G x} / / H=\operatorname{dim} \overline{G e} / / H$. As we have seen in the proof of Lemma $5.3, \operatorname{tr} \cdot \operatorname{deg} \mathbb{k}[G y]^{H}=\operatorname{tr} \cdot \operatorname{deg} \mathbb{k}[\overline{G y}]^{H}$ for each orbit. This settles (1).

By [AP14, Prop. 2.9], we have $\mathbb{k}(G y)^{H}=\operatorname{Quot}\left(\mathbb{k}[G y]^{H}\right)$ for each orbit. Hence the dimension of a generic $H$-orbit on $G y$ is equal to $\operatorname{dim}(G y)-\operatorname{tr} \cdot \operatorname{deg} \mathbb{k}[G y]^{H}$. Thus, (1) implies (2).

The dimension of $\mu(G y)$ is equal to the dimension of a generic $H$-orbit on $G y$, see (5.2). Therefore it does not change along a sheet either.

The defect of a Hamiltonian action can be expressed via the corank

$$
\operatorname{def}(G y)=\operatorname{dim}(G y)-\max _{y^{\prime} \in G y} \operatorname{dim}\left(H y^{\prime}\right)-\operatorname{cork}\left(G y^{\prime}\right) .
$$

In view of (3) and Theorem 5.4, the defect does not change, $\operatorname{def}(G x)=\operatorname{def}(G e)$.

Finally, $\max _{\xi \in \mu(G y)} \operatorname{dim}(H \xi)=\operatorname{dim} \mu(G y)-\operatorname{def}(G y)$, see (5·3).

Of course, there are examples such that $\mu(G x) \neq \mu(G e)$.

Example 5.6. Consider $(\mathfrak{g}, \mathfrak{h})=\left(\mathfrak{s l}_{3}, \mathfrak{s l}_{2}\right)$ and take $x=\operatorname{diag}(1,1,-2)$. Then $e$ is a minimal nilpotent element. Here $\mu(G x)$ is the $\mathrm{SL}_{2}$-orbit of $\operatorname{diag}(1,-1)$, and $\mu(G e)$ is the null-cone in $\mathfrak{s l}_{2}$. We have $\operatorname{dim}(G x)=4$ and $\operatorname{dim} \mu(G x)=\operatorname{dim} \mu(G e)=2$. Further, $\boldsymbol{b}(\mu(G x))=1=$ $\boldsymbol{b}(\mu(G e))$. The $\mathrm{SL}_{2}$-action on $G x$ and on $G e$ has corank 1. 
Example 5.7. Take $G=\mathrm{GL}_{3}, H=\mathrm{GL}_{2}, x=\operatorname{diag}(2,2,1)$. Here the $H$-action on each $G y \subset \mathfrak{g}^{*}$ is coisotropic. We have

$$
\mu(G x)=\bigcup_{a \in \mathbb{k}} H\left(\begin{array}{ll}
2 & 0 \\
0 & a
\end{array}\right) \cup H\left(\begin{array}{ll}
2 & 1 \\
0 & 2
\end{array}\right) .
$$

Further, $e$ is conjugate to $E_{12}$ in $\mathfrak{s l}_{3}$ and

$$
\mu(G e)=\bigcup_{a \in \mathbb{k}} H\left(\begin{array}{ll}
0 & 0 \\
0 & a
\end{array}\right) \cup H\left(\begin{array}{ll}
0 & 1 \\
0 & 0
\end{array}\right) .
$$

5.2. The orthogonal case. There are the orthogonal versions of the Gelfand-Tsetlin subalgebra and the $\lambda$-system of Guillemin-Sternberg. Suppose that $\mathfrak{g}=\mathfrak{s o}_{n}=\mathfrak{s o}_{n}(\mathbb{k})$. Fix a sequence

$$
\mathfrak{s o}_{n} \supset \mathfrak{s o}_{n-1} \supset \ldots \supset \mathfrak{s o}_{3} \supset \mathfrak{s o}_{2} .
$$

Let $\mathcal{C} \subset \mathcal{S}(\mathfrak{g})$ be the subalgebra generated by $\mathcal{S}\left(\mathfrak{s o}_{m}\right)^{\mathfrak{s o}_{m}}$ with $n \geqslant m \geqslant 2$. Then $\mathcal{C}$ is the image in $\mathcal{S}(\mathfrak{g})$ of the famous commutative GT-subalgebra of $\mathcal{U}(\mathfrak{g})$ [GT50']. Hence $\{\mathcal{C}, \mathcal{C}\}=$ 0 . Similar to the $\mathfrak{g l}_{n}$ case, $\mathcal{C}$ has $\boldsymbol{b}(\mathfrak{g})$ algebraically independent generators. Comparing Poincaré series one can prove that in the orthogonal case, the GT-subalgebra $C$ cannot be realised as a limit of MF-subalgebras. Nevertheless, our results in [PY18, Sect. 6.2] show that $\mathcal{C}$ is a maximal Poisson-commutative subalgebra of $\mathcal{S}(\mathfrak{g})$.

With the obvious changes, one defines strongly regular and strongly nilpotent elements, as well as the $\lambda$-system related to eigenvalues. In the orthogonal case, there are no strongly nilpotent elements $e$ such that $\operatorname{dim} d_{e} \mathcal{C}=\boldsymbol{b}(\mathfrak{g})$ if $n \geqslant 4$, see [CE18, Prop. 5.14]. Theorem 4.17 of that paper asserts that $\mathcal{C}$ is complete on each regular coadjoint orbit. We prove that $\mathcal{C}$ is complete on each coadjoint orbit, lifting the assumption that the orbit is regular.

Theorem 5.8. For any $x \in \mathfrak{s o}_{n}$, the GT-subalgebra $\mathcal{C} \subset \mathcal{S}\left(\mathfrak{s o}_{n}\right)$ is complete on every (co)adjoint orbit $\mathrm{SO}_{n} x$ and the action of $\mathrm{SO}_{n-1}$ on $\mathrm{SO}_{n} x$ is coisotropic.

Proof. Assume that both statements are true for $\mathrm{SO}_{n-1}$. The base of induction is the case $n=2$, where the assertions are obvious.

Since $(G, H)=\left(\mathrm{SO}_{n}, \mathrm{SO}_{n-1}\right)$ is a strong Gelfand pair, the action of $\mathrm{SO}_{n-1}$ on $G / B$ is spherical, see Remark 3.4. Hence the action of $\mathrm{SO}_{n-1}$ on $T^{*}(G / B)$ and its image under the moment map $\mu: T^{*}(G / B) \rightarrow \mathfrak{g}^{*}$ is coisotropic, see Section 3 and [AP14, Sect. 2.3]. The regular nilpotent orbit $G \boldsymbol{e} \subset \mathfrak{g}^{*}$ is dense in this image. Therefore the action of $\mathrm{SO}_{n-1}$ on $G \boldsymbol{e}$ is coisotropic, cf. [AP14, Thm 2.6]. The same can be said about any Richardson orbit. However, not every nilpotent orbit in $\mathfrak{s o}_{n}$ is Richardson.

The unique sheet containing $G \boldsymbol{e}$ is $\mathfrak{g}_{\mathrm{reg}}^{*}$. By Lemma 5.2, $\operatorname{cork}_{H}(\mathcal{O})=0$ for each $\mathcal{O} \subset \mathfrak{g}_{\mathrm{reg}}^{*}$. Theorem 5.4 extends this fact to all orbits, cf. [AP14, Prop. 2.7]. 
Next we need to go through the standard inductive argument used, for example, in [GS83']. Let $\mathcal{C}^{[1]}$ be the Gelfand-Tsetlin subalgebra in $\mathcal{S}(\mathfrak{h})$ and $\mathcal{C}^{[2]}-$ in $\mathcal{S}\left(\mathfrak{s o}_{n-2}\right)$. Set $Y=\overline{\mu(G x)}$. Each fibre of the quotient map $Y \rightarrow Y / / H$ contains an open orbit. Therefore $\operatorname{dim} Y / / H=\operatorname{dim} Y-r$, where $r=\max _{y \in Y} \operatorname{dim}(\mathfrak{h} y)$. Hence also $\operatorname{dim} d_{y}\left(\mathcal{S}(\mathfrak{h})^{\mathfrak{h}}\right)=\operatorname{dim} Y-r$ for generic $y \in Y$. By induction, $\mathrm{e}^{[1]}$ is complete on each $H y \subset Y$. More precisely, $\mathrm{e}^{[2]}$ is complete on $H y$. Since $\mathcal{S}(\mathfrak{h})^{\mathfrak{h}} \subset \mathcal{C}^{[1]}$, we have

$$
\operatorname{dim} d_{y} \mathrm{e}^{[1]}=(\operatorname{dim} Y-r)+\frac{r}{2}=\boldsymbol{b}(Y)
$$

for generic $y \in Y$. Since the action of $H$ on $G x$ is coisotropic, we have $\boldsymbol{b}(Y)=\frac{1}{2} \operatorname{dim}(G x)$ by (5.4) and thereby $\mathrm{C}^{[1]}$ is complete on $G x$. Thus, $\mathcal{C}$ is complete on $G x$.

\section{REFERENCES}

[AP02] D. AKHIEZER and D. PANYUSHeV. Multiplicities in the branching rules and the complexity of homogeneous spaces, Mosc. Math. J., 2 (2002), no. 1, 17-33.

[AP14] R.S. Avdeev and A.V. Petukhov. Spherical actions on flag varieties, Mat. Sb., 205 (2014), no. 9, 3-48; English translation in Sb. Math., 205 (2014), no. 9-10, 1223-1263.

[B91] A. BolsinOv. Commutative families of functions related to consistent Poisson brackets, Acta Appl. Math., 24, no. 3 (1991), 253-274.

[BZ16] A. BolsinOv and P. ZHANG. Jordan-Kronecker invariants of finite-dimensional Lie algebras, Transform. Groups, 21 (2016), no. 1, 51-86.

[BK79] W. BORHO and H. KRAFT. Über Bahnen und deren Deformationen bei linearen Aktionen reduktiver Gruppen, Comment. Math. Helv. 54 (1979), no. 1, 61-104.

[CM10] J.-Y. ChARbONNEL and A. MOREAU. The index of centralizers of elements of reductive Lie algebras, Doc. Math., 15 (2010), 387-421.

[CE18] M. COLARUSSO and S. EvENS. The complex orthogonal Gelfand-Zeitlin system, arxiv: $1808.04424 \mathrm{v} 1$ [math.RT].

[CRR] P. CROOKS, S. ROSEMANN, and M. ROESER. Slodowy slices and the complete integrability of Mishchenko-Fomenko subalgebras on regular adjoint orbits, arxiv:1803.04942v1 [math.SG].

[DZ05] J.-P. DufOuR and N.T. ZunG. "Poisson structures and their normal forms". Progress in Mathematics, 242. Birkhäuser Verlag, Basel, 2005.

[FFR10] B. FEIGIN, E. FRENKEL, and L. RYBNIKOV. Opers with irregular singularity and spectra of the shift of argument subalgebra, Duke Math. J., 155 (2010), no. 2, 337-363.

[GT50] I.M. GELFAND and M.L. Tsetlin. Finite-dimensional representations of the group of unimodular matrices. (Russian) Doklady Akad. Nauk SSSR (N.S.) 71(1950), 825-828. English transl. in: I.M. GELFAND, Collected Papers, vol. II, Springer-Verlag, Berlin, 1988, pp. 653-656.

[GT50'] I.M. GELFAND and M.L. Tsetlin. Finite-dimensional representations of groups of orthogonal matrices. (Russian) Doklady Akad. Nauk SSSR (N.S.) 71 (1950), 1017-1020. English transl. in: I.M. Gelfand, Collected Papers, vol. II, Springer-Verlag, Berlin, 1988, pp. 657-661.

[dG08] W. DE GRAAF. Computing with nilpotent orbits in simple Lie algebras of exceptional type, LMS J. Comput. Math., 11 (2008), 280-297.

[GS80] V. Guillemin and S. Sternberg. The moment map and collective motion, Ann. Physics, 127 (1980), no. 1, 220-253. 
[GS83] V. Guillemin and S. SternberG. The Gelfand-Cetlin system and quantization of the complex flag manifolds, J. Funct. Anal., 52 (1983), no. 1, 106-128.

[GS83'] V. GUILLEMIN and S. STERnBERG. On collective complete integrability according to the method of Thimm, Ergodic Theory Dynam. Systems, 3 (1983), no. 2, 219-230.

[GS84] V. Guillemin and S. Sternberg. Multiplicity-free spaces, J. Differential Geom., 19(1984), no.1, 31-56.

[H06] M. HARADA. The symplectic geometry of the Gelfand-Cetlin-Molev basis for representations of $\operatorname{Sp}(2 n, \mathbb{C})$, J. Symplectic Geom., 4 (2006), no. 1, 1-41.

[H82] G.J. Heckman. Projections of orbits and asymptotic behavior of multiplicities for compact connected Lie groups, Invent. Math., 67 (1982), no. 2, 333-356.

[HW90] A.T. Huckleberry and T. Wurzbacher. Multiplicity-free complex manifolds, Math. Ann., 286 (1990), no. 1-3, 261-280.

[J01] K.D. Johnson. A note on branching theorems, Proc. Amer. Math. Soc., 129 (2001), no. 2, 351-353.

[Kn90] F. KNOP. Der Zentralisator einer Liealgebra in einer einhüllenden Algebra, J. Reine Angew. Math., 406 (1990), 5-9.

[Kn90'] F. KNOP. Weylgruppe und Momentabbildung, Invent. Math., 99 (1990), 1-23.

[K63] B. KostAnT. Lie group representations on polynomial rings, Amer. J. Math., 85 (1963), 327-404.

[K09] B. Kostant. Fomenko-Mischenko theory, Hessenberg varieties, and polarizations, Lett. Math. Phys., 90 (2009), no. 1-3, 253-285.

[KW06] B. Kostant and N. WALlaCH. Gelfand-Zeitlin theory from the perspective of classical mechanics. I., Studies in Lie theory, 319-364, Progr. Math., 243, Birkhäuser Boston, Boston, MA, 2006.

[Kr76] M. KRÄMER. Multiplicity free subgroups of compact connected Lie groups, Arch. Math., 27 (1976), 28-36.

[K76] H. KRAFT. Parametrisierung von Konjugationsklassen in $\mathfrak{s l}_{n}$. Math. Ann. 234 (1978), no. 3, 209-220.

[Lo09] I.V. LosEV. Algebraic Hamiltonian actions, Math. Z., 263 (2009), no. 3, 685-723.

[L72] D. LuNA. Sur les orbites fermées des groupes algébriques réductifs, Invent. Math., 16 (1972), 1-5.

[MF78] A.S. MisHCHENKO and A.T. FOMENKO. Euler equation on finite-dimensional Lie groups, Math. USSR, Izv. 12 (1978), 371-389.

[MY17] A. MOLEV and O. YAKIMOVA. Quantisation and nilpotent limits of Mishchenko-Fomenko subalgebras, arxiv:1711.03917v1 [math.RT], $32 \mathrm{pp}$.

[P94] D. PANYUShev. Complexity and nilpotent orbits, Manuscripta Math., 83 (1994), 223-237.

[P03] D. PANYUSHEV. The index of a Lie algebra, the centraliser of a nilpotent element, and the normaliser of the centraliser, Math. Proc. Camb. Phil. Soc., 134, Part 1 (2003), 41-59.

[PPY] D. PAnYushev, A. Premet and O. YAKIMOVA. On symmetric invariants of centralisers in reductive Lie algebras, J. Algebra, 313 (2007), 343-391.

[PY08] D. PANYUSHEV and O. YAKIMOVA. The argument shift method and maximal commutative subalgebras of Poisson algebras, Math. Res. Letters, 15, no. 2 (2008), 239-249.

[PY18] D. PANYUSheV and O. YAKIMOVA. Poisson-commutative subalgebras of $\mathcal{S}(\mathfrak{g})$ associated with involutions, arXiv: 1809.00350v1 [math.RT], 34 pp.

[T02] A.A. TARASOV. The maximality of certain commutative subalgebras in the Poisson algebra of a semisimple Lie algebra, Russian Math. Surveys 57 (2002), no. 5, 1013-1014.

[T91] R.C. THOMPSON. Pencils of complex and real symmetric and skew matrices, Linear Algebra and its Appl., 147 (1991), 323-371. 
[Vi91] E.B. VINBERG. Some commutative subalgebras of a universal enveloping algebra, Math. USSR-Izv. 36 (1991), 1-22.

[Vi01] E.B. VINBERG. Commutative homogeneous spaces and co-isotropic symplectic actions, Uspekhi Mat. Nauk, 56 (2001), no. 1(337), 3-62; English translation in Russian Math. Surveys, 56 (2001), no. 1, 1-60.

[VK78] E.B. VINBERG and B.N. KIMELFELD. Homogeneous domains on flag manifolds and spherical subgroups of semisimple Lie groups, Funktsional. Anal. i Prilozhen., 12(1978), no.3, 12-19; English translation in Functional Anal. Appl., 12 (1978), no. 3, 168-174.

[VP89] Э.Б. ВИнБеРГ, В.Л. Попов. “Теория Инвариантов”, В: Соврем. пробл. математики. Фундаментальные направл., т. 55, стр. 137-309. Москва: ВИНИТИ 1989 (Russian). English translation: V.L. POPOV and E.B. VINBERG. "Invariant theory", In: Algebraic Geometry IV (Encyclopaedia Math. Sci., vol. 55, pp.123-284) Berlin Heidelberg New York: Springer 1994.

[VY18] E.B. VINBERG and O.S. YAKIMOVA. Complete families of commuting functions for coisotropic Hamiltonian actions, arXiv:math/0511498 [math.SG], v3, dated 30.XII.18, 15pp.

[Y06] O. YAKIMOVA. The centralisers of nilpotent elements in classical Lie algebras, Funct. Anal. Appl. 40, no. 1 (2006), 42-51.

[Y09] O. YAKIMOVA. Surprising properties of centralisers in classical Lie algebras, Ann. Inst. Fourier (Grenoble) 59 no. 3 (2009), 903-935.

[Z09] A.A. ZORIN. On the commutativity of the centralizer of a subalgebra in a universal enveloping algebra, Funktsional. Anal. i Prilozhen., 43 (2009), no. 2, 47-63; English translation in Funct. Anal. Appl., 43 (2009), no. 2, 119-131.

(D. Panyushev) Institute for Information Transmission Problems of the R.A.S., Bolshoi KARETNYI PER. 19, MOSCOW 127051, RUSSIA

E-mail address: panyushev@iitp.ru

(O. Yakimova) UniVersitët ZU KÖLN, MATHEMATISCHES INSTITUt, WeYERTAL 86-90, 50931 KÖLN, DEUTSCHLAND

E-mail address: yakimova.oksana@uni-koeln.de 